\title{
A plate motion study using Ajisai SLR data
}

\author{
Arata Sengoku \\ Hydrographic Department, Maritime Safety Agency, 5-3-1, Tsukiji, Chuo, Tokyo 104-0045, Japan
}

(Received July 28, 1997; Revised May 11, 1998; Accepted May 12, 1998)

\begin{abstract}
Eight years of Ajisai SLR data were processed to determine the terrestrial reference frame and its time evolution. The typical precision and accuracy of the estimated geocenter position averaged over a year determined from a oneyear Ajisai SLR data set are $1 \mathrm{~cm}$ and $1.5 \mathrm{~cm}$, respectively. The baselines between SLR stations away from plate boundaries show rates of change that are in good agreement with NUVEL-1A, ITRF93 and LAGEOS results but significant deviations from geologically determined plate motion models are found for stations in plate boundary regions. Velocities of the observation stations were estimated by a weighted least squares method. The Simosato SLR station, located $100 \mathrm{~km}$ away from the plate boundary between the Eurasian plate and the Philippine Sea plate, moves in the direction of the subduction of the Philippine Sea plate with respect to the Eurasian plate, which infers strong coupling of the two plates at the boundary. The motion of other stations at plate boundary regions is also discussed. This study is the first attempt to use Ajisai SLR data to determine the global terrestrial reference frame and its variation, thus independent of the previous SLR studies most of which were based on LAGEOS SLR analyses.
\end{abstract}

\section{Introduction}

Until the 1970s, present-day plate motion had been estimated from geological data only. At the end of the 1970s, the U.S. National Aeronautics and Space Administration (NASA) initiated the Crustal Dynamics Project (CDP) to measure plate motion by using space geodetic techniques such as Satellite Laser Ranging (SLR) and Very Long Baseline Interferometry (VLBI). In the late 1980s, Global Positioning System (GPS) became a highly promising geodetic technique for crustal deformation studies mainly thanks to the rapid technological development of GPS receivers and the constellation of satellites.

\subsection{Plate motion studies by SLR}

In 1979, Smith et al. (1979) reported the first result of baseline change obtained from SLR to BEACON EXPLORER-C. They estimated that the distance between two laser stations in California was decreasing at a rate $50 \%$ larger than the geological prediction (Minster and Jordan, 1978). The SLR experiment was called SAFE (San Andreas Fault Experiment), the first attempt to detect crustal deformation by using space geodetic techniques. The CDP started in 1979 to apply space methods to further our understanding of Earth dynamics. Tapley et al. (1985) analyzed over 22,000 baseline estimates from 60-day subsets of eight-year LAGEOS observation data set; LAGEOS is a geodetic satellite launched by the U.S. in 1976. Good agreement between geologically predicted and observed plate motion was found, especially for the North American and Pacific stations. Christodoulidis et al. (1985) used LAGEOS data from 1979 to 1982 to recover chord rates between SLR

Copy right (C) The Society of Geomagnetism and Earth, Planetary and Space Sciences (SGEPSS); The Seismological Society of Japan; The Volcanological Society of Japan; The Geodetic Society of Japan; The Japanese Society for Planetary Sciences. stations. The correlation coefficient between the SLR observed and geologically predicted tectonic rates among the 34 pairs of SLR stations was 0.64. Other geodetic, geodynamic and geophysical results from LAGEOS SLR data were also published in the LAGEOS special issue of Journal of Geophysical Research in 1985. Harrison and Douglas (1990) used the least squares procedure to calculate velocities of SLR stations from over 130 baseline rates. They found significant deviation from the geologic means for Quincy, Huahine and Simosato. Smith et al. (1990) estimated station velocities from ten years LAGEOS SLR data in a solution called SL7. Using 12 stations, centrally located on five tectonic plates, they found a high correlation of 0.989 between their SLR estimates and NUVEL-1 (DeMets et al., 1990). The estimated error of site velocities was a few $\mathrm{mm} /$ year. Watkins (1990) showed baseline rates from 12.5 years of LAGEOS SLR data. Robbins et al. (1993) showed that SLR and VLBI observations across plate boundaries are 6.3\% slower than NUVEL-1. Robaudo and Harrison (1993) determined the Euler vectors of plate motion from SLR and VLBI results. Discrepancies with NUVEL-1, that is, the difference between plate motion averaged over millions of years and several years, are generally within the errors of the geodetic estimates.

Scientists have tried to reveal the plate boundary tectonics. In the Mediterranean region, the WEGENER-MEDLAS project started in 1985. Transportable SLR systems have been deployed in the central and eastern Mediterranean Sea region. Wilson and Reinhart (1993), Cenci et al. (1993), Zerbini (1993) and Sinclair and Appleby (1993) estimated site velocities of these stations. Noomen et al. (1993) showed that the motions of the stations in southern Italy and southern France indicate deformation consistent with the African/ Eurasian plate boundary. In Asia, the JHD determined terrestrial reference frames (Sengoku, 1991) and tectonic 
motion with LAGEOS SLR data by using the original software HYDRANGEA (Sasaki, 1990). They found significant deviation of Simosato movements from the plate motion models (Sasaki and Sengoku, 1993).

\subsection{Plate motion studies by VLBI and GPS}

VLBI began in the late 1960s as a tool for radio astronomy, but it was recognized that baseline vector between ground stations could be determined with a very high precision as a by-product. Routine geodetic VLBI observation in North America began at the beginning of the 1980s. Over 110 VLBI stations had participated in geodetic VLBI observation until 1993 (Boucher et al., 1994). Several global VLBI networks, such as IRIS (International Radio Interferometric Surveying), have been managed for Earth rotation monitoring and for geodetic studies by NASA, the U.S. National Geodetic Survey (NGS), the National Astronomical Observatory (NAO) of Japan, and so on. NASA has deployed mobile VLBI systems in the western U.S.A. for the study of deformation along the plate boundary between the North American and the Pacific plates (Gordon et al., 1993). Site velocities near the San Andreas Fault indicate large scale deformation patterns existing within a broad zone between the two plates. Ryan et al. (1993a) showed that movement of stations well away from plate boundaries is continuous and smooth. They also found that internal stability of the North American plate is better than $2 \mathrm{~mm} /$ year. The GSFC/NASA has released annual analysis results of geodetic VLBI (Ryan et al., 1993b) which have been used in studies of regional crustal deformation (Argus and Lyzenga, 1993; Feigl et al., 1993) and global tectonics (Lisowski, 1991; Fallon and Dillinger, 1992). Saucier and Humphreys (1993) combined VLBI results with geological slip rates and fault distribution to model the long term horizontal deformation in southern California.

GPS was developed in the early 1970 s in the U.S.A. as a military navigation system. It became fully operational in 1993 with 24 satellites in six orbital planes. The first international GPS geodetic campaign, CASA-UNO, was carried out in 1988 in North and South America for regional tectonic investigations. Today, seven analysis centers of the International GPS Service for Geodynamics (IGS) produce daily global solutions for the IGS network that consists of more than 96 permanent GPS stations. The purpose of IGS is to provide precise GPS ephemerides and to support regional GPS observations. Using GPS data spanning more than 3.5 years, Dixon (1991) estimated a spreading rate across the Gulf of California, which agrees well with NUVEL-1A. Larson and Freymueller (1995) estimated the Euler vectors of plate motion including the Antarctic plate from global GPS data. They found a good agreement with NUVEL-1A within estimated errors. Tsuji (1995) estimated day-by-day motions of four GPS sites in Japan and compared them to the plate motion models. GPS is the most prevalent space geodetic technique at present.

Today, GPS is widely used for regional tectonic studies. GPS is logistically advantageous for most geoscientists because of its portability and low price. The role of SLR and VLBI in plate motion studies is maintenance of the terrestrial reference frame with accurate origin (geocenter) and orientation. These techniques support regional GPS networks for connection to global networks. SLR also contributes to geophysics, especially geopotential determination, its variations, ocean tide modeling and atmospheric density studies.

\subsection{Ajisai}

Ajisai is the first Japanese geodetic satellite launched on August 12, 1986 from the Tanegashima Space Center by the National Space Development Agency (NASDA). The body of Ajisai is a hollow sphere covered with 1436 corner cube reflectors (CCR's) for SLR tracking and 318 mirrors for photography.

SLR observations of Ajisai have been conducted at the global SLR tracking network since its launch, and more than 8 years of tracking data have been accumulated. One reason for the SLR tracking activity to the Ajisai satellite by the international community is because the altitude of Ajisai is close to that of TOPEX/POSEIDON. Tracking of Ajisai has contributed to the recovery of the geopotential field that is indispensable for the precision orbit determination of TOPEX/POSEIDON(Lerch et al., 1992; Tapley et al., 1994). Thanks to significant improvements of the geopotential models in recent years, the precision of orbit determination of Ajisai has improved as well, which makes Ajisai an effective tool for the determination of the global terrestrial reference frame and its time variation. Ajisai is especially effective for the position determination of weak SLR systems with small laser power and with small aperture of receiving telescope.

In this paper, the terrestrial reference frame and its time evolution are discussed from eight years of Ajisai SLR data. This is the first attempt to determine the global terrestrial reference frame and its variation by using this Japanese geodetic satellite. The most terrestrial reference frame studies have used LAGEOS because of its high altitude. Our results are independent of LAGEOS observation models and force models. Therefore, this study validates the SLR terrestrial reference frames previously determined by LAGEOS tracking data.

\section{Analysis of SLR Data and Geodetic Parameter Determination}

The solution for Ajisai ephemerides, dynamic model parameters and geodetic parameters was obtained using the University of Texas orbit analysis system, UTOPIA (McMillan, 1973; Tapley et al., 1985). This data analysis system implements a weighted least squares batch procedure which estimates the initial conditions for a numerical solution of the differential equation governing the satellite motion. A modified Encke method was used in the numerical integration process of the UTOPIA to accomplish high accuracy and stability. The integration step size for Ajisai was 30 seconds. In the UTOPIA processing, the dynamical equations were expressed in the geocentric, nonrotating cartesian coordinate system defined by the mean equator and the equinox of epoch 2000.0 (J2000.0). The UTOPIA used in this study was version 9012 implemented on a CRAY Y-MP8/864 supercomputer at the University of Texas Center for High Performance Computing. 
2.1 Force models, measurement models and reference frames

Force models

The force models that were used in the analysis of an eight-year Ajisai SLR data set are described in this section. All models are included in the University of Texas UTOPIA software system.

The force models used in UTOPIA are mostly based on the IERS Standards (McCarthy, 1992) with several exceptions (Table 1). The nonspherical gravitational force of the Earth is by far the largest perturbing force acting on Ajisai. The JGM-3 gravity model (Tapley et al., 1994), complete up to degree and order 70, was used for the Earth gravitational force. The gravitational constant, GM, was fixed to $398600.4415 \mathrm{~km}^{3} / \mathrm{s}^{2}$. Gravitational attractions of the Moon, the Sun and the seven major planets were included. The mass ratios of these celestial bodies to the Earth are given in the IERS Standards. DE-200 (Newhall et al., 1983) was used as the ephemeris source for the celestial bodies. The dynamic effect of ocean tides was modeled by Cheng et al. (1993) derived from satellite tracking data. The solid Earth tide model was based on the IERS Standards but expanded to include third and fourth degree terms (Eanes and Watkins, 1994). The relativistic perturbation was also included following Ries et al. (1988).

Four types of surface forces, which are proportional to the area to mass ratio, were included in the analysis of Ajisai. These forces were the atmospheric drag, the solar radiation pressure, the Earth albedo and infrared radiation from the Earth. The modeling of atmospheric drag is one of the most difficult components to handle in the Ajisai orbit analysis since the precision of the atmospheric density calculation at the Ajisai altitude is poor. Frequent estimation of the atmospheric drag coefficient, $C_{\mathrm{d}}$, is the most effective way to remove errors in the atmospheric density model. The Drag Temperature Model (DTM) (Barlier et al., 1978) was adopted in the analysis since its accuracy is supposed to be superior to other models for Ajisai (Cheng, private communication, 1994). One component of radiation pressure, the anisotropic reflection model (Sengoku et al., 1995) was used for the solar radiation pressure force. The other components included were associated with the Earth. Earth's albedo effect is caused by the radiation reflected or scattered as short-wave radiation at the Earth's surface. The infrared radiation of the Earth is thermally re-emitted from the Earth's surface and somewhat "pushes" the satellite. Photon thrusts by these surface forces were numerically modeled by Knocke and Ries (1987) and implemented in UTOPIA.

\section{Measurement models}

The SLR measurement is the two-way travel time that a laser pulse requires to travel from the station to the satellite and back to the station. Though the measurement is based on simple principles, it requires several corrections to obtain the distance from the observation station to the satellite. The tropospheric delay of an optical wavelength signal is dominated by the dry term. The troposphere wet term and the ionospheric term are less than one centimeter, therefore, are smaller than the measurement uncertainties in most cases. The dry term is approximated by Marini and Murray (1973) with an accuracy of less than $1 \mathrm{~cm}$ at 20 degrees elevation. The center of mass correction is the range difference between the CCR that reflects the laser light and the center of mass of the satellite. The average value of the center of mass correction is designed to be $1.01 \mathrm{~m}$ for Ajisai (Sasaki and Hashimoto, 1987). The position of the tracking stations in an Earth-centered reference frame is changing due to solid Earth tides and ocean loading. These effects are well known and formulated in the IERS Standards. The site displacement due to atmospheric loading is neglected in this paper since it is smaller than tidal displacements. Note that the correction due to solid Earth tides includes a permanent term, i.e., the station coordinate values are not equal to the mean value. The relativistic propagation delay due to the Earth's gravitation is also considered (Holdridge, 1967).

\section{Reference frames}

The equations of motion of Ajisai were numerically integrated in the geocentric, inertial reference frame of J2000.0. This system is realized by the JPL DE-200 planetary ephemeris, the 1976 International Astronomical Union(IAU) precession (Lieske et al., 1977) and the 1980 IAU Wahr nutation models (Wahr, 1981). Corrections to the VLBIdetermined IAU precession and nutation models were also applied. The transformation between the Earth-fixed and true-of-date reference frames was carried out by using the Greenwich mean sidereal time (Aoki et al., 1982) and the EOP(CSR)94L01 Earth orientation series determined by

Table 1. Adopted force models and measurement models for Ajisai orbit analysis.

\begin{tabular}{|c|c|c|}
\hline Item & Model and value & Reference \\
\hline Geopotential & JGM-3 $(70 \times 70)$ & Tapley et al., 1994 \\
\hline Solid Earth tide (dynamic effect) & IERS Standards, expanded to third and fourth degree & Eanes and Watkins, 1994 \\
\hline Ocean tide model (dynamic effect) & Cheng et al. (1993) & Cheng et al., 1993 \\
\hline GM & $398600.4415 \mathrm{~km}^{3} / \mathrm{s}^{2}$ & IERS Standards, 1992 \\
\hline Relativistic effect (dynamical effect) & IERS Standards, one body & Ries et al., 1988 \\
\hline Atmospheric density & DIM & Barlier et al., 1978 \\
\hline Radiation pressure of the Sun & Anisotropic reflection model & Sengoku et al., 1995 \\
\hline Earth's albedo & UTOPIA model & Knocke and Ries, 1987 \\
\hline Infrared radiation of the Earth & UTOPIA model & Knocke and Ries, 1987 \\
\hline Tropospheric delay & IERS Standards & Marini and Murray, 1973 \\
\hline Center of mass & $1.01 \mathrm{~m}$ & Sasaki and Hashimoto, 1987 \\
\hline Solid Earth and ocean loading (kinematic effect) & IERS Standards & IERS Standards, 1992 \\
\hline Relativistic effect (propagation delay) & IERS Standards & Holdridge, 1967 \\
\hline
\end{tabular}


LAGEOS SLR data (Eanes and Watkins, 1994).

To define a terrestrial reference frame from analyses based on satellite techniques, it is necessary to constrain, at least, three parameters if EOP's are estimated. Other constraints are usually imposed to define the terrestrial reference frame as the Earth center of mass, or geocenter. One robust way to define the terrestrial reference frame is to constrain the latitude of a station and the latitude and longitude of another station whose longitude is 90 degrees apart from the former site. Furthermore, the time evolution of the position of these defining stations has to be stable and well known. The author selected Greenbelt and Maui as the defining stations since they are located well away from the plate boundaries and their motions are close to the geological predictions (Robbins et al., 1993). The latitude of Greenbelt and the latitude and longitude of Maui were fixed to the SSC(CSR)94L01r02 (Eanes, private communication, 1994) determined by LAGEOS SLR data by the CSR/UT. The velocities of these sites were fixed to the NUVEL-1 plate motion model.

\subsection{Solution strategies}

The primary purpose of this study is to demonstrate the capability of the Ajisai satellite for geodesy and geodynamics. Generally, the accuracy of geodetic results from satellite tracking data depends on the altitude of the satellite. Higher satellites' orbits are simpler to model since the effects of higher harmonics of the geopotential field are sufficiently small or even negligible. On the other hand, the quality of lower satellites' orbits usually suffers from errors in the geopotential and atmospheric density models, even though the accuracy of the geopotential field models is about one order of magnitude better than a decade ago. For example, in LAGEOS orbit computation, the geopotential field coefficients are truncated at degree and order 20, while a more complete set of coefficients has to be considered in the Ajisai orbit estimation. Therefore, appropriate solution strategies are required to obtain an Ajisai solution that has accuracy comparable to that of LAGEOS.

Single-and multi-arc methods

There are two kinds of solution strategies commonly used in the analysis of satellite tracking data: the single- and multi-arc methods.

The single-arc, or long-arc, method fits a single dynamical consistent trajectory of a satellite over the whole period. The errors in the applied dynamical models, truncation and round-off errors of the numerical integrator make the result less accurate. Typical residual RMS's for a one-year Ajisai SLR data set and a fifteen-year LAGEOS set are about $1 \mathrm{~m}$ and $1.3 \mathrm{~m}$, respectively (Cheng, private communication, 1995). This means that the precision of the estimated orbit is lower than these numbers, and the accuracy of the estimated geodetic parameters is poor. Clearly, the long-arc method is not appropriate for geodynamic studies unless adequate modeling is introduced to accommodate the model errors. However, the long-arc is required for the study of the dynamical models used in the analysis. The differences between Kepler elements estimated by the single-arc method and those derived from the multi-arc method contain precious information on the model errors of the geopotential field, ocean tides and surface forces.

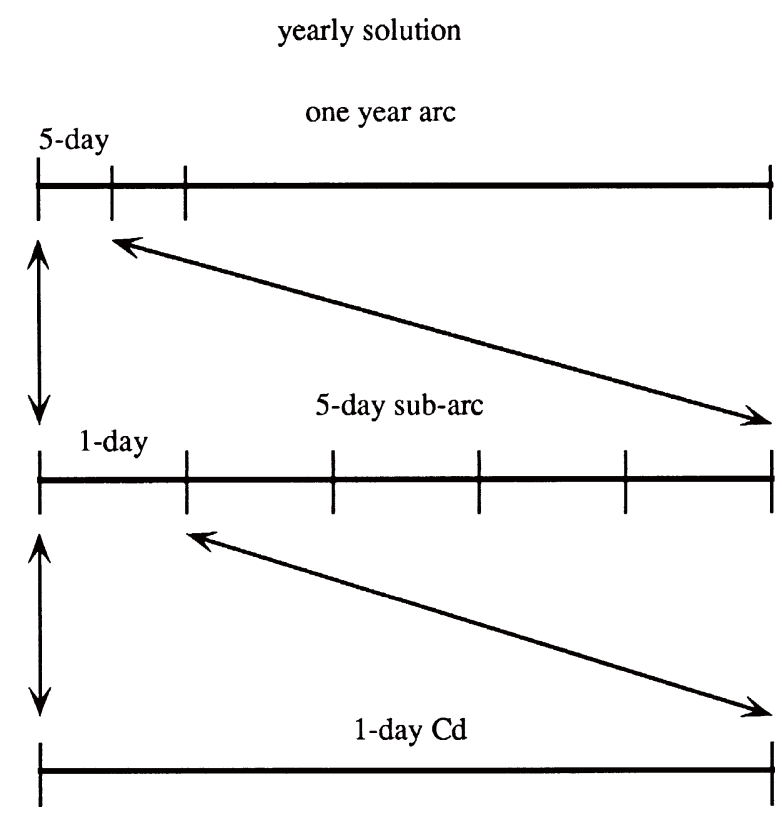

Fig. 1. Schematic representation of the solution procedure adopted for this study. A one-year arc was divided into 5-day sub-arcs. Arc parameters were estimated once 5 days. The atmospheric drag coefficient, $C_{\mathrm{d}}$, was estimated once a day. Global parameters were solved once a year.

The multi-arc, or short-arc, method divides the whole arc into short arcs. For the most part, the long-periodic effects due to the applied model errors are removed from the residuals since they are accommodated in the estimated initial position and velocity of the satellite and other parameters. The global parameters include station coordinates which are considered to be nearly constant for a longer time scale. Note that the velocities of stations are assumed to be zero, they are estimated afterward (Section 3). The multi-arc approach is suitable for the geodynamic study. However, we have to note that some geophysical signals may be lost in this approach.

The multi-arc method was adopted in this study for precise terrestrial reference frame determination. The author adopted a 5-day sub-arc for the analysis since it is empirically known to be stable. The residual RMS's for 5-day arcs are close to the observation noise. The global parameters were estimated once per one-year arc. The one-year global arc was adopted for safe and economical operation of UTOPIA (Fig. 1).

\section{Arc and global parameters}

The Ajisai orbit must be fitted as precisely as possible to obtain high quality estimates of station coordinates. Empirical periodic accelerations in the radial and normal directions were introduced whose periods correspond to the orbital period of Ajisai. This type of empirical acceleration is very effective in removing orbit errors. The initial state vector of the satellite, the radial and normal components of once per revolution accelerations, and the Earth Orientation Parameters (EOP), $x_{\mathrm{p}}$ and $y_{\mathrm{p}}$, were estimated every 5 days. The author estimated the atmospheric drag coefficient, $C_{\mathrm{d}}$, once a day.

Consequently, the adopted analysis procedure in the next section was: 
Table 2. Summary of Ajisai SLR data used in this study.

\begin{tabular}{lrrrrrrrrr}
\hline & 1986 & 1987 & 1988 & 1989 & 1990 & 1991 & 1992 & 1993 & 1994 \\
\hline Stations & 16 & 22 & 21 & 30 & 31 & 34 & 38 & 35 & 30 \\
Passes & 1067 & 2088 & 2587 & 2963 & 2479 & 2652 & 4634 & 4442 & 2504 \\
NP* & 19084 & 35256 & 46456 & 49877 & 39699 & 54661 & 76525 & 73555 & 42680 \\
\hline
\end{tabular}

*NP: normal point.

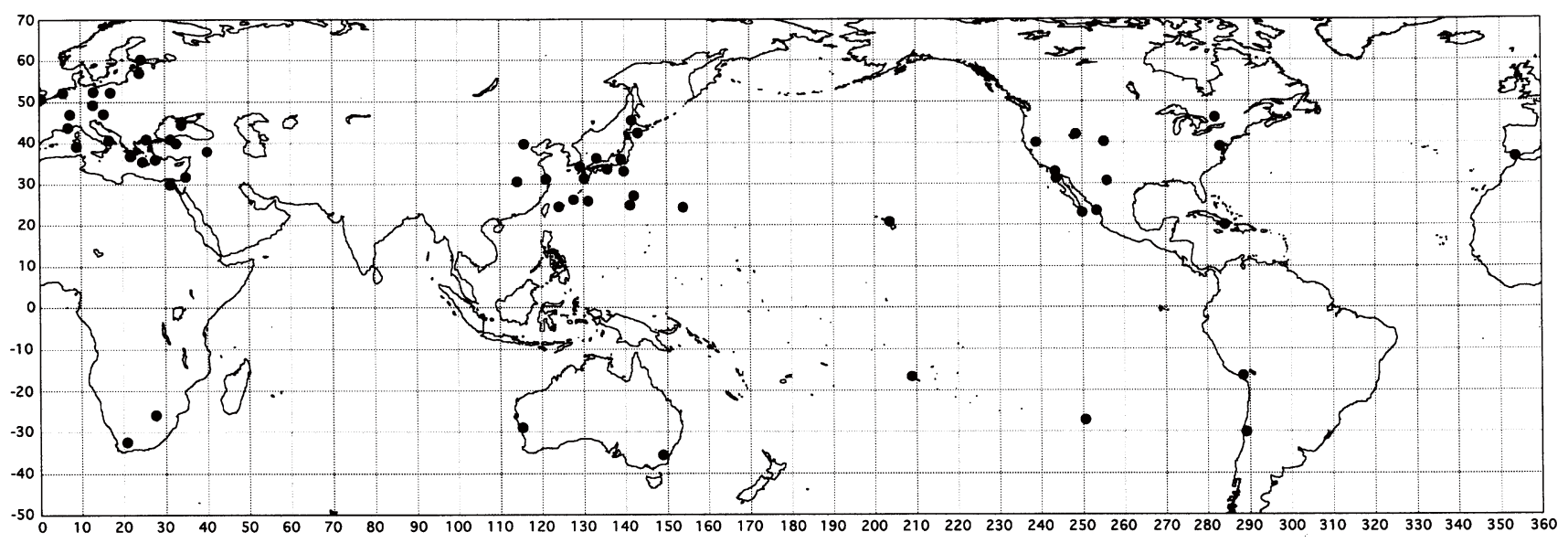

Fig. 2. Distribution of Ajisai tracking stations.

1) 5-day arcs of Ajisai SLR data were converged with the estimation of the following arc parameters: the initial position and velocity of Ajisai, the radial and normal components of once per revolution acceleration, the Earth orientation $\left(x_{\mathrm{p}}, y_{\mathrm{p}}\right)$ and daily $C_{\mathrm{d}}$,

2) a one-year arc was generated by combining the short arcs. Station coordinates and reflectivity coefficients were solved as global parameters, i.e., a single set of parameters for the one-year arc.

\section{Eight-Year Analysis of Ajisai SLR Data}

Ajisai has been continuously tracked by global SLR tracking network since the launch. Until June 1994, more than 20,000 Ajisai passes were obtained at 68 stations on 8 major plates. The author analyzed the eight-year Ajisai SLR data in order to precisely determine the terrestrial reference frame and to estimate the station velocity.

\subsection{Data description}

SLR tracking data of Ajisai were compressed into 30second normal points (NP's) following the Herstmonceux recommendation that was outlined at the Fifth International Workshop on Laser Ranging Instrumentation in 1984 (Watkins, 1990). Some data were judged as outliers and were omitted for the analysis if they appeared to have large range bias or time bias values.

Table 2 shows yearly statistics of the Ajisai NP's. The Ajisai SLR data analyzed in this paper span the period from 12 August 1986 to 31 June 1994. Until June 1994, 68 stations participated in the observation of Ajisai (Fig. 2). In Japan, there were two permanent sites, Simosato and Dodaira and 13 temporary sites that observed using the transportable HTLRS (Sengoku, 1997). Figures 3 and 4 show the history of the number of stations that have observed Ajisai and the number of NP's in the 5-day arcs. Both the numbers of stations and NP's have been increasing since 1992.

The quality of SLR data in the 1970s when the first SLR measurements were made was a few meters. This quality has been steadily improved since then. In 1986, when Ajisai was launched, NASA had third generation SLR stations in operation and their precision was $1 \mathrm{~cm}$ or less. Other SLR stations also had comparable quality except for several second generation stations in operation. The a priori sigma of SLR data obtained by the third generation systems was assumed to be $10 \mathrm{~cm}$ in this paper. The quality of SLR data depends upon the hardware systems and quality control of the tracking data at the tracking stations. This kind of quality variation can be observed in the preliminary analysis for the generation of NP's as a station dependent noise level. Hence, a priori sigma's of SLR data for stations with poor quality were set to values larger than $10 \mathrm{~cm}$ to stabilize the solution.

\subsection{Stability of terrestrial reference frames determined by Ajisai one-year arc analysis}

Eight years of Ajisai normal point data were analyzed with the multi-arc approach using the UTOPIA analysis program. Residual RMS's after the fit were mostly constant for the eight years (Fig. 5). This uniform data quality is important for reliable estimation of station velocity. Slightly larger residual RMS's were observed in 1986, which may be caused by inappropriate control of observation conditions at tracking sites such as excessively high voltage at the detectors or threshold levels. A hump in 1989 and 1990 can be explained by the higher solar activity. The atmospheric density in this period was more than 10 times higher than 


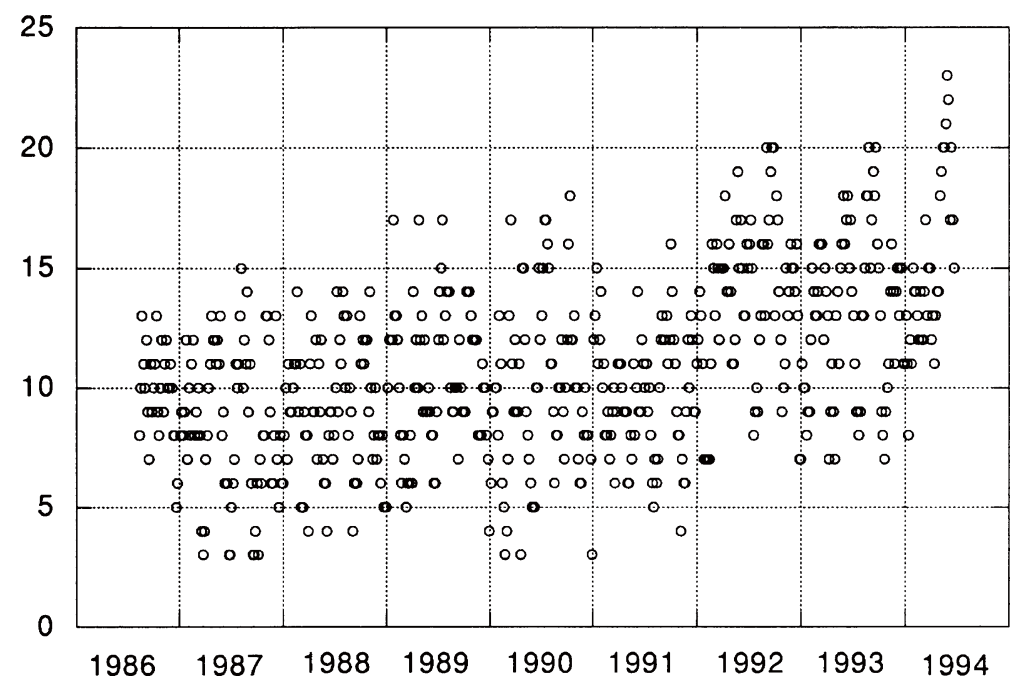

Fig. 3. Number of Ajisai tracking stations in 5-day interval from August 1986 to June 1994.

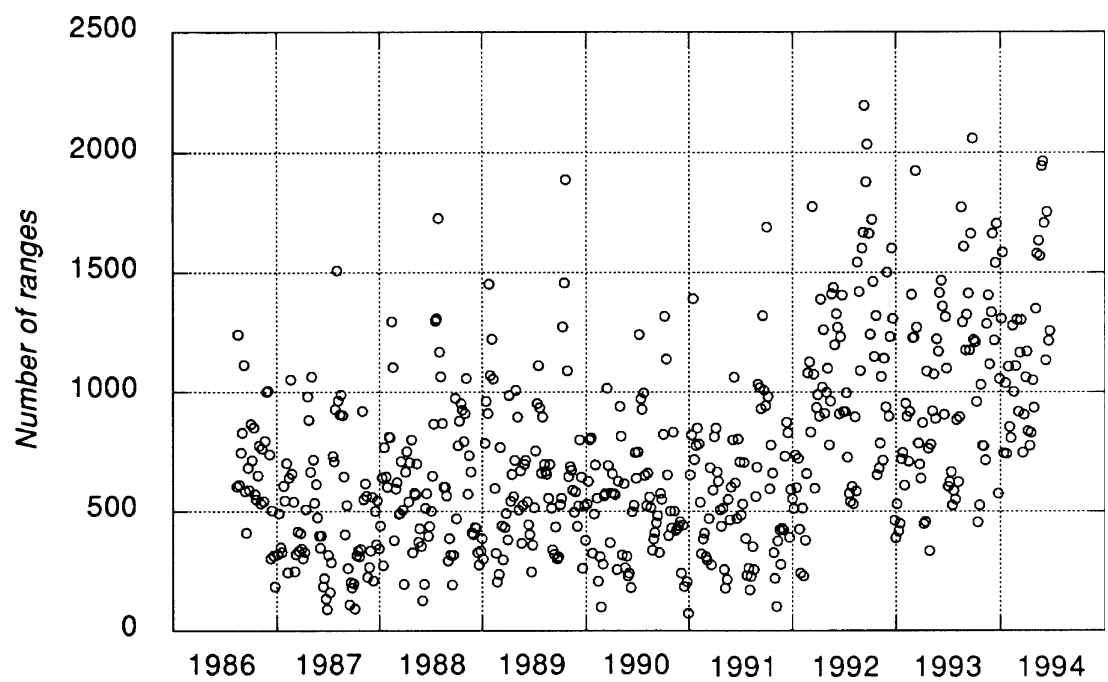

Fig. 4. Number of Ajisai normal points in 5-day interval from August 1986 to June 1994.

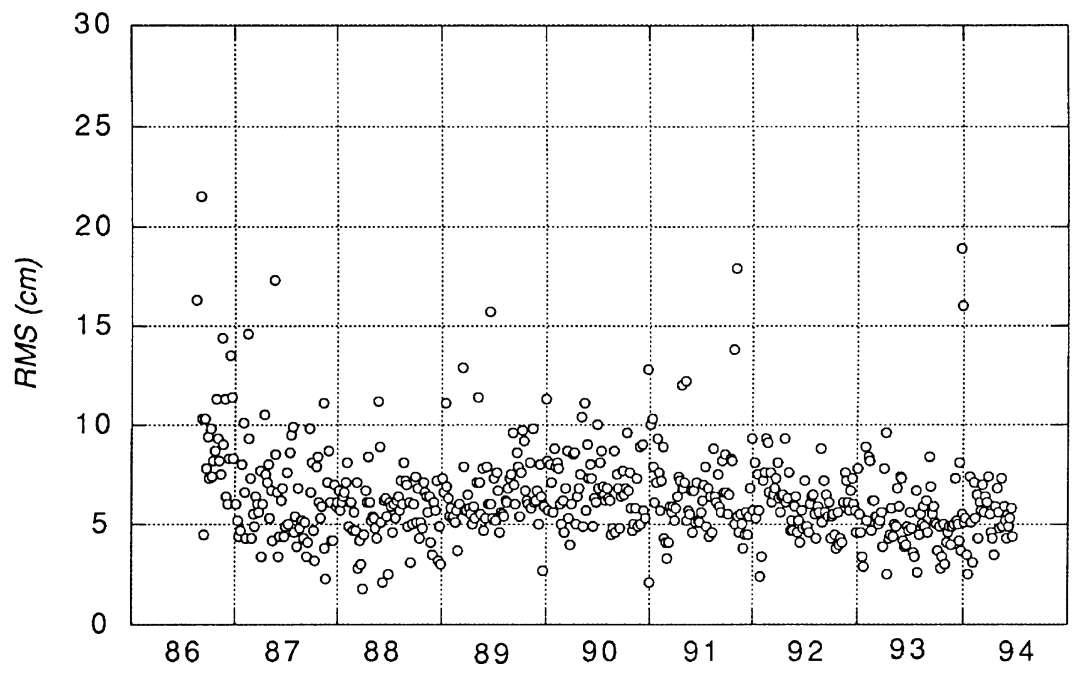

Fig. 5. Residual RMS's of Ajisai SLR data after the fit in 5-day interval from August 1986 to June 1994. 
Table 3. Seven-parameter transformation from TRFxx to TRF93 (unit: $d X, d Y, d Z(\mathrm{~cm}), s\left(10^{-9}\right), r x, r y, r z$ (milli-arcsecond)).

\begin{tabular}{|c|c|c|c|c|c|c|c|}
\hline & $d X$ & $d Y$ & $d Z$ & $s$ & $r x$ & $r y$ & $r z$ \\
\hline TRF86 & $1.0 \pm 1.9$ & $1.9 \pm 2.0$ & $1.7 \pm 1.8$ & $-1.3 \pm 2.8$ & $0.9 \pm 0.8$ & $1.2 \pm 0.7$ & $-0.5 \pm 0.6$ \\
\hline TRF87 & $-0.3 \pm 0.9$ & $0.8 \pm 1.0$ & $-1.6 \pm 0.9$ & $-3.0 \pm 1.4$ & $-0.1 \pm 0.4$ & $-0.8 \pm 0.4$ & $1.0 \pm 0.3$ \\
\hline TRF88 & $0.2 \pm 0.9$ & $1.3 \pm 0.9$ & $0.0 \pm 0.8$ & $-1.4 \pm 1.3$ & $0.8 \pm 0.4$ & $0.2 \pm 0.3$ & $0.5 \pm 0.3$ \\
\hline TRF89 & $1.0 \pm 0.7$ & $0.1 \pm 0.7$ & $-1.0 \pm 0.6$ & $-0.7 \pm 1.0$ & $-0.6 \pm 0.3$ & $-0.4 \pm 0.3$ & $0.8 \pm 0.2$ \\
\hline TRF90 & $2.0 \pm 1.2$ & $1.6 \pm 1.3$ & $-3.5 \pm 1.2$ & $-0.6 \pm 1.8$ & $-2.9 \pm 0.5$ & $0.1 \pm 0.5$ & $4.2 \pm 0.4$ \\
\hline TRF91 & $1.0 \pm 0.6$ & $0.9 \pm 0.6$ & $-1.1 \pm 0.6$ & $-0.7 \pm 0.9$ & $-0.3 \pm 0.3$ & $-0.4 \pm 0.2$ & $-0.6 \pm 0.2$ \\
\hline TRF92 & $-0.4 \pm 0.4$ & $0.0 \pm 0.5$ & $0.3 \pm 0.4$ & $0.0 \pm 0.7$ & $0.2 \pm 0.2$ & $0.0 \pm 0.2$ & $-0.2 \pm 0.2$ \\
\hline TRF93 & - & - & - & - & - & - & - \\
\hline TRF94 & $-0.4 \pm 0.5$ & $0.3 \pm 0.5$ & $-1.0 \pm 0.5$ & $-0.8 \pm 0.7$ & $-0.1 \pm 0.2$ & $0.2 \pm 0.2$ & $-0.1 \pm 0.2$ \\
\hline Mean & $0.5 \pm 0.9$ & $0.9 \pm 0.7$ & $-0.7 \pm 1.5$ & $-1.1 \pm 0.9$ & $-0.3 \pm 1.2$ & $0.0 \pm 0.6$ & $0.6 \pm 1.6$ \\
\hline Mean* & $0.3 \pm 0.7$ & $0.8 \pm 0.7$ & $-0.4 \pm 1.1$ & $-1.1 \pm 0.9$ & $0.1 \pm 0.6$ & $0.0 \pm 0.6$ & $0.1 \pm 0.6$ \\
\hline
\end{tabular}

*A mean without TRF90.

Table 4. Seven-parameter transformation from TRFxx to SSC(CSR)93L01r2 (unit: $d X, d Y, d Z(\mathrm{~cm}), s\left(10^{-9}\right), r x, r y, r z(\mathrm{milli}-\operatorname{arcsecond})$ ).

\begin{tabular}{|c|c|c|c|c|c|c|c|}
\hline & $d X$ & $d Y$ & $d Z$ & $s$ & $r x$ & $r y$ & $r z$ \\
\hline TRF86 & $-0.4 \pm 2.0$ & $0.1 \pm 2.1$ & $-1.7 \pm 1.9$ & $6.1 \pm 2.9$ & $-0.4 \pm 0.8$ & $-1.6 \pm 0.8$ & $0.4 \pm 0.7$ \\
\hline TRF87 & $0.8 \pm 1.1$ & $1.3 \pm 1.1$ & $1.7 \pm 1.0$ & $7.7 \pm 1.6$ & $0.7 \pm 0.5$ & $0.5 \pm 0.4$ & $-1.1 \pm 0.4$ \\
\hline TRF88 & $0.3 \pm 1.0$ & $0.7 \pm 1.0$ & $0.0 \pm 0.9$ & $6.1 \pm 1.5$ & $-0.2 \pm 0.4$ & $-0.6 \pm 0.4$ & $-0.6 \pm 0.3$ \\
\hline TRF89 & $-0.5 \pm 1.1$ & $1.9 \pm 1.1$ & $1.1 \pm 1.0$ & $5.4 \pm 1.6$ & $1.2 \pm 0.5$ & $0.1 \pm 0.4$ & $-0.8 \pm 0.4$ \\
\hline TRF90 & $-1.5 \pm 1.5$ & $0.4 \pm 1.6$ & $3.5 \pm 1.4$ & $5.3 \pm 2.2$ & $3.5 \pm 0.7$ & $-0.4 \pm 0.6$ & $-4.3 \pm 0.5$ \\
\hline TRF91 & $-0.5 \pm 1.0$ & $1.1 \pm 1.1$ & $1.1 \pm 1.0$ & $5.4 \pm 1.5$ & $0.9 \pm 0.4$ & $0.1 \pm 0.4$ & $0.6 \pm 0.4$ \\
\hline TRF92 & $0.9 \pm 1.0$ & $2.0 \pm 1.1$ & $-0.3 \pm 1.0$ & $4.7 \pm 1.5$ & $0.4 \pm 0.4$ & $-0.4 \pm 0.4$ & $0.8 \pm 0.4$ \\
\hline TRF93 & $0.5 \pm 1.0$ & $2.0 \pm 1.0$ & $0.0 \pm 0.9$ & $4.7 \pm 1.4$ & $0.6 \pm 0.4$ & $-0.4 \pm 0.4$ & $-0.1 \pm 0.3$ \\
\hline TRF94 & $0.9 \pm 1.1$ & $1.7 \pm 1.2$ & $1.0 \pm 1.1$ & $5.5 \pm 1.7$ & $0.7 \pm 0.5$ & $-0.6 \pm 0.4$ & $0.0 \pm 0.4$ \\
\hline Mean & $0.1 \pm 0.8$ & $1.2 \pm 0.7$ & $0.7 \pm 1.5$ & $5.7 \pm 0.9$ & $0.8 \pm 1.1$ & $-0.4 \pm 0.6$ & $-0.6 \pm 1.5$ \\
\hline Mean* & $0.3 \pm 0.6$ & $1.4 \pm 0.7$ & $0.4 \pm 1.1$ & $5.7 \pm 1.0$ & $0.5 \pm 0.5$ & $-0.4 \pm 0.6$ & $-0.1 \pm 0.7$ \\
\hline
\end{tabular}

*A mean without TRF90.

that during a low solar activity period. The residual RMS's were sometimes large when Ajisai data were not sufficient to provide a robust solution in a five-day arc.

Stability of terrestrial reference frames determined by Ajisai

The TRF determined from 19xx Ajisai arc is indicated as TRFxx hereafter. For example, TRF87 is the terrestrial reference frame determined from the 1987 one-year arc.

The quality of the obtained TRF's was estimated through internal and external consistency checks. The internal consistency check compares the TRF's determined from Ajisai data. The comparison with the LAGEOS result (SSC(CSR)93L01r2) is called the external consistency check in this paper.

Internal consistency check

A transformation from TRF 1 to TRF 2 is generally performed by the following seven-parameter formula (Bursa's model):

$$
\left(\begin{array}{l}
X \\
Y \\
Z
\end{array}\right)_{2}=\left(\begin{array}{l}
X \\
Y \\
Z
\end{array}\right)_{1}-\left(\begin{array}{l}
d X \\
d Y \\
d Z
\end{array}\right)-\left(\begin{array}{ccc}
s & -r_{z} & r_{y} \\
r_{z} & s & -r_{x} \\
-r_{y} & r_{x} & s
\end{array}\right)\left(\begin{array}{l}
X \\
Y \\
Z
\end{array}\right)_{1},
$$

where $d X, d Y$ and $d Z$ are difference of the origins, $s$ stands for scale difference and $r_{x}, r_{y}$ and $r_{z}$ represent the rotation around the $x, y$ and $z$ axes, respectively. Each TRF determined by the one-year arc was transformed to TRF93, which is regarded as stable due to the large number of normal points. These parameters were estimated by a least squares procedure using positions of 10 sites that were commonly observed in every one-year arc, i.e., Grasse, Graz, Greenbelt, Maui, Matera, Monument Peak, Quincy, Simosato, Yaragadee and Zimmerwald. The author assumed equal weight for every coordinate component. Noted that we have only one station in the southern hemisphere which may increase errors in $d Z, r_{x}$ and $r_{y}$.

As is shown in Table 3, the typical difference in origin is $1 \mathrm{~cm}$ except for TRF90, which means that the Ajisai oneyear arc can define the geocenter with a precision of $1 \mathrm{~cm}$. Note that uncertainties in the following tables stand for one sigma values. In 1990, there is a large origin difference, which may be caused by poor data distribution of Ajisai SLR data. It is also suggested by relatively large value for the standard deviation. The typical difference in orientation is sub milli-arcsecond (mas), which is larger by a factor of two than the origin difference. The scale difference is typically $1 \times 10^{-9}$, which is comparable to the origin difference. 
External consistency check

Table 4 shows the comparison between the TRF's determined by Ajisai and the LAGEOS result, defined as SSC(CSR)93L01r2. Significant differences can be found in $d Y$ and $s$, though the defining stations are the same in these two results. The scale difference is persistent and corresponds to $3.6 \mathrm{~cm}$ in vertical component at Earth's surface. Therefore, the height determined in this study is systematically inconsistent with the LAGEOS result. This may be caused by the error in center of mass correction, or range biases in some tracking stations.

\section{Velocities of SLR Stations Estimated from Ajisai Eight-Year SLR Data and Their Geophysical Interpretation}

In this section, change rates of arc lengths between SLR stations were estimated through the weighted least squares method. Velocities of stations were estimated from eight years of Ajisai SLR data and were compared to geologically derived plate motion models and other results obtained from space geodetic techniques.

4.1 Arc length rate estimates from yearly solutions of Ajisai SLR data

As described in the previous sections, Ajisai SLR data were analyzed year by year through the multi-arc approach, and nine yearly solutions of station coordinates from 1986 to 1994 were derived. The author combined these solutions to determine velocities of stations by the weighted least squares procedure. One method to estimate velocities of stations from time series of terrestrial reference frames is to use "arc length rates".

The arc length is a product of the angular distance between stations and equatorial radius (Fig. 6). It is commonly observed that estimated positions of stations contain errors caused by inconsistency of the origin, orientation and scale of the terrestrial reference frames. Hence, velocities of stations can be poorly recovered from change rates determined from station positions (Smith et al., 1991). Arc length rates are free from errors due to the orientation and scale differences of the terrestrial reference frames, but are definitely affected by the origin difference. The maximum arc length error due to origin inconsistency is twice as large as the origin difference (Smith et al., 1991). Origin inconsistency can be removed by adjusting the geocenter difference of the TRF's. The stability of the TRF's is a key in the study of station velocity estimation. Chord rates, change rates of straight distance between stations, are free from orientation inconsistency, but are not independent of scale inconsistency. Geodesic distance rates, change rates of station distance along the reference ellipsoid, are dependent on the origin, orientation and scale inconsistency, though they are the geodetically fundamental definition of distance between stations.

The arc length, $l$, is the length of an arc between two stations along the sphere with equatorial radius, $a_{\mathrm{e}}$;

$$
\begin{aligned}
l & =a_{\mathrm{e}} \psi_{\mathrm{g}} \\
& =a_{\mathrm{e}} \cos ^{-1}\left(\sin \varphi_{1} \sin \varphi_{2}+\cos \varphi_{1} \cos \varphi_{2} \cos \left(\lambda_{1}-\lambda_{2}\right)\right),
\end{aligned}
$$

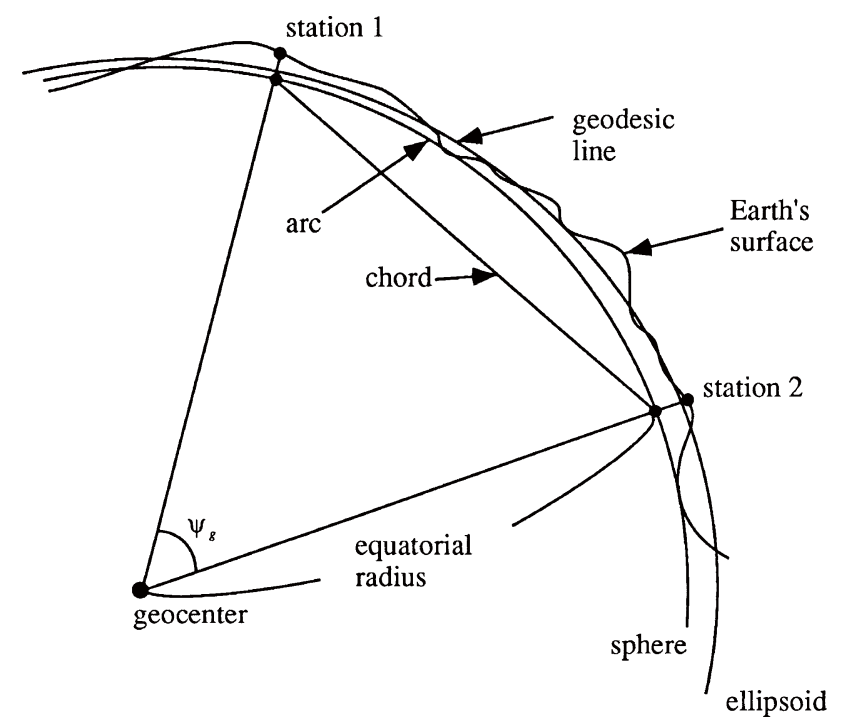

Fig. 6. Definition of arc, chord and geodesic line.

where $\psi_{\mathrm{g}}$ is the geocentric angle between the stations (Fig. 6), $\varphi$ and $\lambda$ stand for geocentric latitude and longitude of a station, respectively. Note that geocentric, not geodetic, latitude and longitude are used, hereafter. The arc length rate reflects horizontal velocities of stations and is independent of vertical velocities. Generally, the arc length rate is determined more precisely than straight distance rates since wellknown error sources of SLR, such as range bias and its variation, mostly affect the vertical component of the sites.

The arc lengths and their errors were calculated from the yearly TRF solutions. The geocenter inconsistency was corrected by transformation parameters determined in the previous section. Let $\sigma_{\lambda_{1} \varphi_{2}}$ be an element of the covariance matrix for $\lambda_{1}$ and $\varphi_{2}$, and vice versa, which are directly estimated in the UTOPIA. Rigorously speaking, the covariance matrix the UTOPIA estimated is referred to geodetic, not geocentric, latitude. However, the difference between covariance matrices for geocentric and geodetic latitude are small and negligible. The estimated error of the arc length, $\sigma_{l}$, is,

$$
\sigma_{l}^{2}=V A V^{T}
$$

where

$$
V=\left(\frac{\partial l}{\partial \varphi_{1}}, \frac{\partial l}{\partial \lambda_{1}}, \frac{\partial l}{\partial \varphi_{2}}, \frac{\partial l}{\partial \lambda_{2}}\right)
$$

$A=\left(\begin{array}{cccc}\sigma_{\varphi_{1} \varphi_{1}} & \sigma_{\varphi_{1} \lambda_{1}} & \sigma_{\varphi_{1} \varphi_{2}} & \sigma_{\varphi_{1} \lambda_{2}} \\ \sigma_{\lambda_{1} \varphi_{1}} & \sigma_{\lambda_{1} \lambda_{1}} & \sigma_{\lambda_{1} \varphi_{2}} & \sigma_{\lambda_{1} \lambda_{2}} \\ \sigma_{\varphi_{2} \varphi_{1}} & \sigma_{\varphi_{2} \lambda_{1}} & \sigma_{\varphi_{2} \varphi_{2}} & \sigma_{\varphi_{2} \lambda_{2}} \\ \sigma_{\lambda_{2} \varphi_{1}} & \sigma_{\lambda_{2} \lambda_{1}} & \sigma_{\lambda_{2} \varphi_{2}} & \sigma_{\lambda_{2} \lambda_{2}}\end{array}\right)$, $\frac{\partial l}{\partial \varphi_{1}}=-\frac{a_{\mathrm{e}}\left\{-\sin \varphi_{1} \cos \varphi_{2} \cos \left(\lambda_{1}-\lambda_{2}\right)+\cos \varphi_{1} \sin \varphi_{2}\right\}}{\sqrt{1-\left\{\cos \varphi_{1} \cos \varphi_{2} \cos \left(\lambda_{1}-\lambda_{2}\right)+\sin \varphi_{1} \sin \varphi_{2}\right\}^{2}}}$, 


$$
\frac{\partial l}{\partial \varphi_{2}}=-\frac{a_{\mathrm{e}}\left\{-\cos \varphi_{1} \sin \varphi_{2} \cos \left(\lambda_{1}-\lambda_{2}\right)+\sin \varphi_{1} \cos \varphi_{2}\right\}}{\sqrt{1-\left\{\cos \varphi_{1} \cos \varphi_{2} \cos \left(\lambda_{1}-\lambda_{2}\right)+\sin \varphi_{1} \sin \varphi_{2}\right\}^{2}}}
$$$$
\frac{\partial l}{\partial \lambda_{1}}=\frac{a_{\mathrm{e}} \cos \varphi_{1} \cos \varphi_{2} \sin \left(\lambda_{1}-\lambda_{2}\right)}{\sqrt{1-\left\{\cos \varphi_{1} \cos \varphi_{2} \cos \left(\lambda_{1}-\lambda_{2}\right)+\sin \varphi_{1} \sin \varphi_{2}\right\}^{2}}},
$$$$
\frac{\partial l}{\partial \lambda_{2}}=-\frac{\partial l}{\partial \lambda_{1}}
$$

The arc length rates were estimated through weighted least squares fitting to arc lengths (see, for example, Press et al., 1986). Arc length rates were estimated for those station pairs with more than four years of observation.

The statistical parameter $\chi^{2}$ was calculated for both cases with and without the geocenter adjustment, that is,

$$
\chi^{2}=\frac{\sum_{i}\left(\left(O_{i}-C_{i}\right) / \sigma_{i}\right)^{2}}{n_{\mathrm{s}}-2}
$$

where $O_{i}$ and $C_{i}$ are the $i$-th observed and calculated arc length, $\sigma_{i}$ is the estimated error of the $i$-th arc length and $n_{\mathrm{s}}$ is the number of solutions for the arc. The mean value of $\chi^{2}$ for all arcs is 0.868 and 1.010 with and without the geocenter adjustment, respectively. The geocenter adjustment gives better and less-scattered arc rates. Furthermore, this implies that the estimated errors of station coordinates in UTOPIA are slightly pessimistic.

\subsection{Comparison of arc rates with other studies}

Table 5 summarizes the arc length rates between the Simosato Hydrographic Observatory (SHO) and other stations. The geologically predicted rates by AM0-2 (Minster and Jordan, 1978) and NUVEL-1 NNR are also shown, assuming that Simosato is on the Eurasian plate. The LAGEOS column represents a velocity field model estimated from LAGEOS SLR data from 1976 to 1994 (SSC(CSR)94L01r02). ITRF represents a combined velocity field model ITRF93, the International Earth Rotation Service Terrestrial Reference Frame '93, produced from SLR, VLBI and GPS analysis results (Boucher et al., 1994). Note that some stations, such as Borowiec and Simeiz, have second generation SLR equipment with poor quality and the estimated rates including these stations are not reliable due to poor stability of the observation equipment. Generally,

Table 5. Arc length rates between Simosato and other stations from Ajisai eight-year analysis (unit: $\mathrm{mm} / \mathrm{year}$ ).

\begin{tabular}{lrrrrrrr}
\hline Arc & Rate & Sigma & AM0-2 & NUVEL & ITRF & LAGEOS & Plate* \\
\hline Simosato-Bar Giyyora & -31 & 9 & -6 & -7 & -36 & & EU-AF \\
Simosato-Helwan & -30 & 9 & -6 & -6 & -34 & -34 & EU-AF \\
Simosato-Matera & -31 & 6 & -3 & -3 & -31 & -31 & EU-AF \\
& & & & & & & \\
Simosato-Orroral & -53 & 37 & -66 & -71 & -58 & & EU-IN/AU** \\
Simosato-Yaragadee & -71 & 6 & -77 & -80 & -71 & -73 & EU-IN/AU** \\
& & & & & & & \\
Simosato-Borowiec & -75 & 57 & 0 & 0 & -23 & & EU-EU \\
Simosato-San Fernando & -61 & 51 & 0 & 0 & & & EU-EU \\
Simosato-Grasse & -26 & 7 & 0 & 0 & -21 & -21 & EU-EU \\
Simosato-Graz & -31 & 7 & 0 & 0 & -27 & -27 & EU-EU \\
Simosato-Potsdam & -41 & 27 & 0 & 0 & -22 & -12 & EU-EU \\
Simosato-Greenwich & -21 & 10 & 0 & 0 & -23 & -24 & EU-EU \\
Simosato-Riga & -64 & 59 & 0 & 0 & -23 & & EU-EU \\
Simosato-Shanghai & -57 & 29 & 0 & 0 & -33 & -43 & EU-EU \\
Simosato-Simeiz & 18 & 70 & 0 & 0 & -25 & & EU-EU \\
Simosato-Wettzell & -24 & 25 & 0 & 0 & -24 & -25 & EU-EU \\
Simosato-Zimmerwald & -20 & 9 & 0 & 0 & -26 & -27 & EU-EU \\
Simosato-Greenbelt & -2 & 7 & -7 & -5 & -5 & -4 & EU-NA \\
Simosato-Mazatlan & -5 & 9 & -12 & -10 & 2 & 4 & EU-NA \\
Simosato-McDonald & 19 & 17 & -11 & -9 & & 2 & EU-NA \\
Simosato-Quincy & -5 & 6 & -11 & -9 & -7 & -7 & EU-NA \\
Simosato-Easter Is. & 89 & 46 & 60 & 56 & 75 & & EU-NZ \\
Simosato-Ensenada & -29 & 20 & -67 & -57 & -42 & -38 & EU-PA \\
Simosato-Huahine & -28 & 73 & -107 & -98 & -69 & & EU-PA \\
Simosato-Mon. Peak & -36 & 6 & -65 & -56 & -37 & -37 & EU-PA \\
Simosato-Maui & -60 & 6 & -99 & -89 & -65 & -63 & EU-PA \\
Simosato-Arequipa & 37 & 17 & -25 & -20 & 7 & 9 & EU-SA \\
\hline & & & & & & & \\
\hline
\end{tabular}

*AF: African plate, AU: Australian plate, EU: Eurasian plate, IN: Indian plate, NA: North American plate, NZ: Nazca plate, PA: Pacific plate, SA: South American plate.

**Indian plate for AM0-2 and Australian plate for NUVEL-1. 


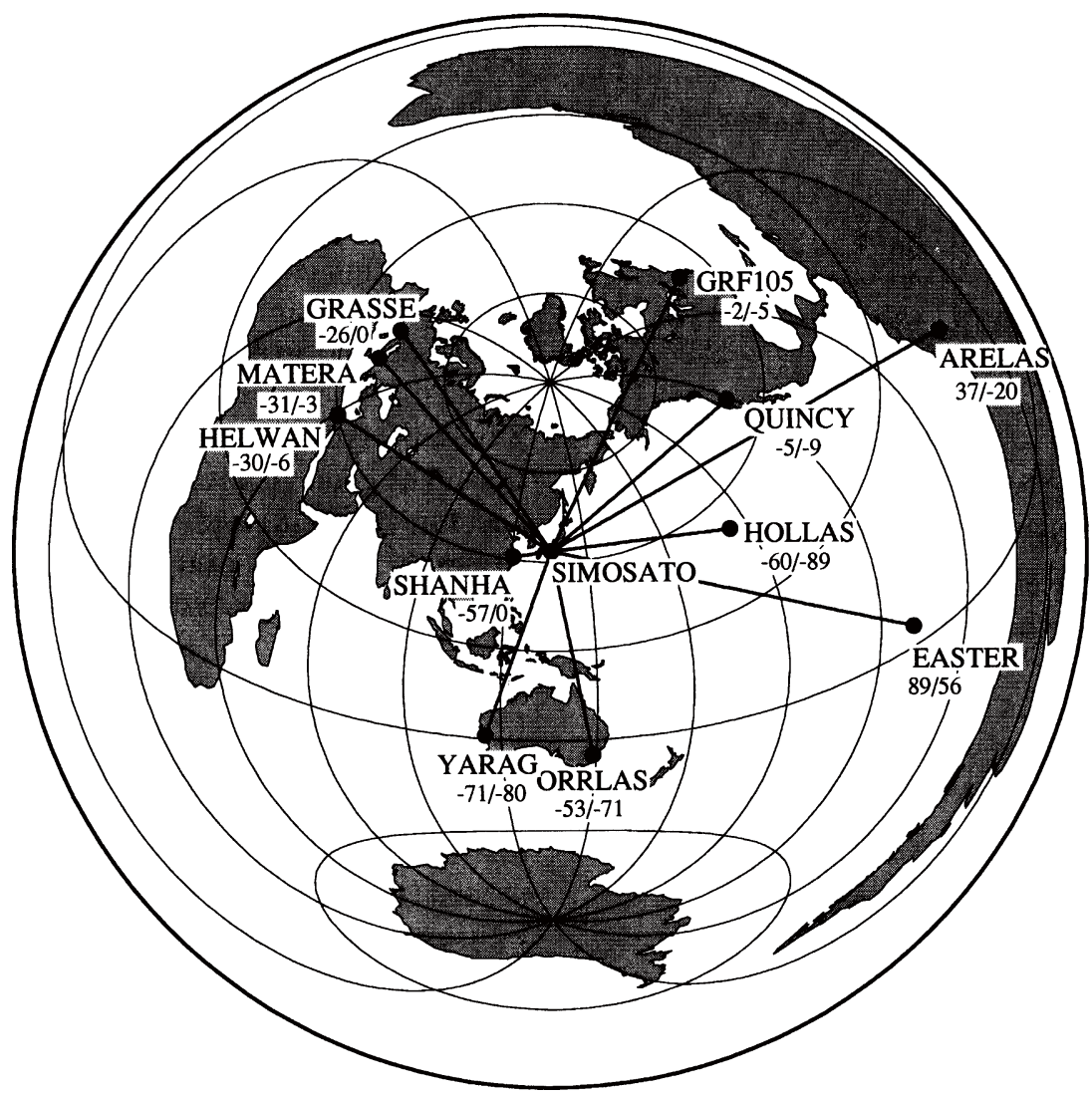

Fig. 7. The azimuthal equidistant projection centered on Simosato showing arc rates determined by Ajisai SLR data (left value) and NUVEL-1 predictions (right value). Unit is $\mathrm{mm} /$ year.

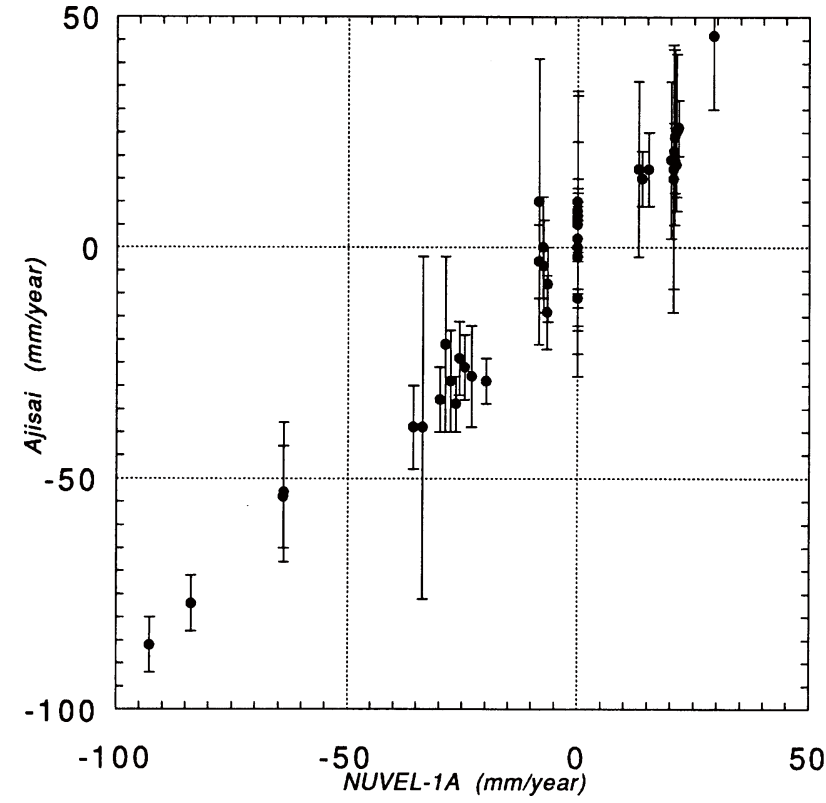

Fig. 8. Correlation plot comparing the arc rates between tracking sites located away from plate boundaries determined from Ajisai eight-year analysis with the rates predicted by NUVEL-1A model. The analysis is based on 42 arcs among 10 sites on five plates. The slope of the best fitting line is $1.007 \pm 0.043$. agreement with the LAGEOS result is fairly good. But significant deviation from the geological models is definitely observed, especially for the European, Pacific and South American stations, which suggests anomalous movement of Simosato with respect to the Eurasian plate. This will be discussed further in the next section. Figure 7 shows baselines including Simosato ( $\mathrm{SHO}$ ) obtained by the azimuthal equidistant projection that preserves distances and azimuths from Simosato to the other stations. This figure was drawn by the GMT software (Wessel and Smith, 1991). Though there is a tight connection to the European stations, the baselines to the southeast and southwest exhibit higher sigma values than the other baselines.

Figure 8 shows the correlation of the arc length rates determined by this study and other velocity models for interplate baselines. Plate boundary stations were omitted to remove the effect of plate boundary deformation. The adopted stations are Helwan, Yaragadee, Grasse, Graz, Greenwich, Wettzell, Zimmerwald, Greenbelt, McDonald and Maui. The associated plates are the African, Australian, Eurasian, North American and Pacific plates. The slope was estimated by weighted least squares fitting of a straight line (Table 6). The Ajisai arc rates agree well with NUVEL-1A (DeMets et al., 1994), ITRF93 and the LAGEOS results (SSC(CSR)94L01r02), but significantly deviate from AM02. Slight deviations in arc rates are observed between this study and NUVEL-1. It is not surprising to find fairly good correlation between the Ajisai and LAGEOS results since the same analysis technique was applied, but it should be 
noted that the analysis strategies and a part of applied measurement/force models are different. The velocities of stations were estimated simultaneously as global parameters in the dynamical analysis of LAGEOS, while they were estimated afterward in this study. This agreement of arc length rates by Ajisai and LAGEOS suggests that our solution strategy is effective and the results are not biased. The geodetic results by Smith et al. (1992) determined from SLR and VLBI data also agree well with NUVEL-1 rates among 20 stations located on the Australian, Eurasian, Nazca, North American and Pacific plates.

4.3 Velocity estimates of stations from arc length rates

To understand on-going plate motion from Ajisai SLR data, a kinematic reference frame (KRF) needs to be defined. In the definition, three parameters must be fixed using a priori velocity information. Though the restrictions on the KRF are arbitrary, the restrictions should be in harmony with the present understanding of plate tectonics for the comparison with other studies. Thus the author fixed the northward and eastward motions of HOLLAS and the northward motion of GRF105 in a manner that is similar to the definition of the terrestrial reference frame. These defining stations provide

Table 6. Correlation of the arc rates by this study and other velocity models.

\begin{tabular}{llccc}
\hline Model & Method & Slope & RMS & Number of arcs \\
\hline AM0-2 & geological & 0.930 & 0.040 & 42 \\
NUVEL-1 & geological & 0.972 & 0.042 & 42 \\
NUVEL-1A & geological & 1.007 & 0.043 & 42 \\
ITRF93 & SLR, VLBI, GPS & 1.000 & 0.045 & 34 \\
CSR & SLR & 1.001 & 0.043 & 42 \\
\hline
\end{tabular}

solution strength since they have sufficient tracking data and are located in the middle of the plate where the motion of the sites is regarded as close to tectonic models (Robbins et al., 1993). These considerations make the KRF robust due to geometrical strength. The author used constant motion of the defining stations given by the NUVEL-1 NNR model. Although our results correlate well with the NUVEL-1A rather than NUVEL-1, the author used NUVEL-1 as a reference because it is more convenient for the comparison with other studies.

Velocities of SLR stations were estimated by the weighted least squares procedures similar to geodetic network adjustments. All arc length rates were combined in a weighted least squares solution that yields the velocity vectors of the sites. The observation equation can be written as follows,

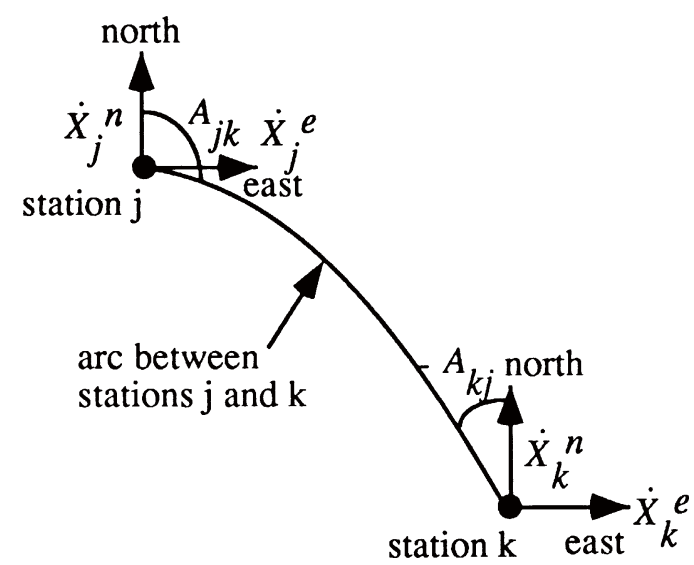

Fig. 9. Azimuth angle and station velocity. Unit of velocity is meter per year.

Table 7. Estimated velocities of SLR stations relative to NUVEL-1 determined from Ajisai eight-year analysis.

\begin{tabular}{|c|c|c|c|c|c|c|c|}
\hline \multirow[t]{2}{*}{ Station } & \multicolumn{2}{|c|}{ Eastward } & \multicolumn{2}{|c|}{ Northwasrd } & \multicolumn{3}{|c|}{ Error ellipse parameters } \\
\hline & $\begin{array}{l}\text { velocity } \\
(\mathrm{mm} / \mathrm{yr})\end{array}$ & $\begin{array}{l}\text { sigma } \\
(\mathrm{mm} / \mathrm{yr})\end{array}$ & $\begin{array}{l}\text { velocity } \\
(\mathrm{mm} / \mathrm{yr})\end{array}$ & $\begin{array}{l}\text { sigma } \\
(\mathrm{mm} / \mathrm{yr})\end{array}$ & $\begin{array}{l}\text { semi-major } \\
(\mathrm{mm} / \mathrm{yr})\end{array}$ & $\begin{array}{l}\text { semi-minor } \\
(\mathrm{mm} / \mathrm{yr})\end{array}$ & $\begin{array}{l}\text { orientation } \\
\quad(\mathrm{deg})\end{array}$ \\
\hline Greenbelt & 1.0 & 3.2 & - & - & - & - & - \\
\hline Grasse & 3.2 & 4.5 & -2.9 & 4.6 & 5.5 & 3.4 & 46 \\
\hline Simosato & -30.3 & 4.3 & 11.5 & 4.4 & 4.8 & 3.6 & 133 \\
\hline Bar Giyyora & -4.2 & 6.1 & -10.9 & 5.3 & 6.9 & 4.2 & 36 \\
\hline Helwan & -5.0 & 6.1 & -8.8 & 5.0 & 6.9 & 3.9 & 35 \\
\hline Orroral & 7.9 & 10.7 & 11.5 & 18.0 & 18.0 & 10.7 & 88 \\
\hline Yaragadee & -9.5 & 3.4 & -4.7 & 4.2 & 4.4 & 3.2 & 114 \\
\hline San Fernando & -6.7 & 15.6 & 31.7 & 22.7 & 22.7 & 15.6 & 92 \\
\hline Graz & 2.1 & 4.7 & 3.6 & 4.5 & 5.5 & 3.5 & 42 \\
\hline Matera & 2.2 & 4.9 & -3.7 & 4.5 & 5.8 & 3.2 & 40 \\
\hline Greenwich & -2.8 & 5.1 & 1.0 & 6.4 & 7.3 & 3.7 & 55 \\
\hline Shanghai & 32.0 & 12.6 & -0.3 & 12.3 & 12.7 & 12.1 & 27 \\
\hline Wettzell & -9.6 & 9.8 & 9.6 & 11.4 & 12.4 & 8.4 & 57 \\
\hline Zimmerwald & -4.1 & 5.0 & -2.4 & 5.4 & 6.3 & 3.7 & 50 \\
\hline Mazatlan & -10.9 & 4.4 & 4.5 & 5.0 & 5.2 & 4.2 & 118 \\
\hline McDonald & 7.9 & 6.2 & -7.5 & 8.2 & 8.4 & 6.0 & 108 \\
\hline Quincy & -10.5 & 3.3 & 5.1 & 4.0 & 4.1 & 3.1 & 112 \\
\hline Easter Is. & 10.5 & 19.8 & 21.9 & 19.8 & 22.4 & 16.8 & 135 \\
\hline Ensenada & -1.0 & 8.8 & -13.6 & 8.8 & 9.9 & 7.5 & 135 \\
\hline Huahine & -25.4 & 39.0 & -90.5 & 29.9 & 42.4 & 24.6 & -29 \\
\hline Mon. Peak & 0.5 & 3.3 & -3.1 & 4.1 & 4.2 & 3.1 & 111 \\
\hline Arequipa & 30.2 & 7.8 & -8.9 & 7.3 & 7.8 & 7.3 & -9 \\
\hline
\end{tabular}


Table 8. Velocity estimates of the Simosato station relative to the NUVEL-1 Eurasian plate motion.

\begin{tabular}{llcc}
\hline References & Technique & $\begin{array}{c}\text { Magnitude } \\
\text { (mm/yr) }\end{array}$ & $\begin{array}{c}\text { Azimuth } \\
\text { (deg) }\end{array}$ \\
\hline Smith et al. (1990) & LAGEOS SLR & 27 & 292 \\
Robaudo and Harrison (1993) & SLR/VLBI & 25 & 296 \\
Eanes and Watkins (1994) & LAGEOS SLR & 29 & 294 \\
Smith et al. (1995) & SLR/VLBIDORIS/GPS & 28 & 303 \\
This study & Ajisai SLR & 32 & 291 \\
Philippine Sea plate motion by Seno et al. (1993) & & 44 & 310 \\
\hline
\end{tabular}

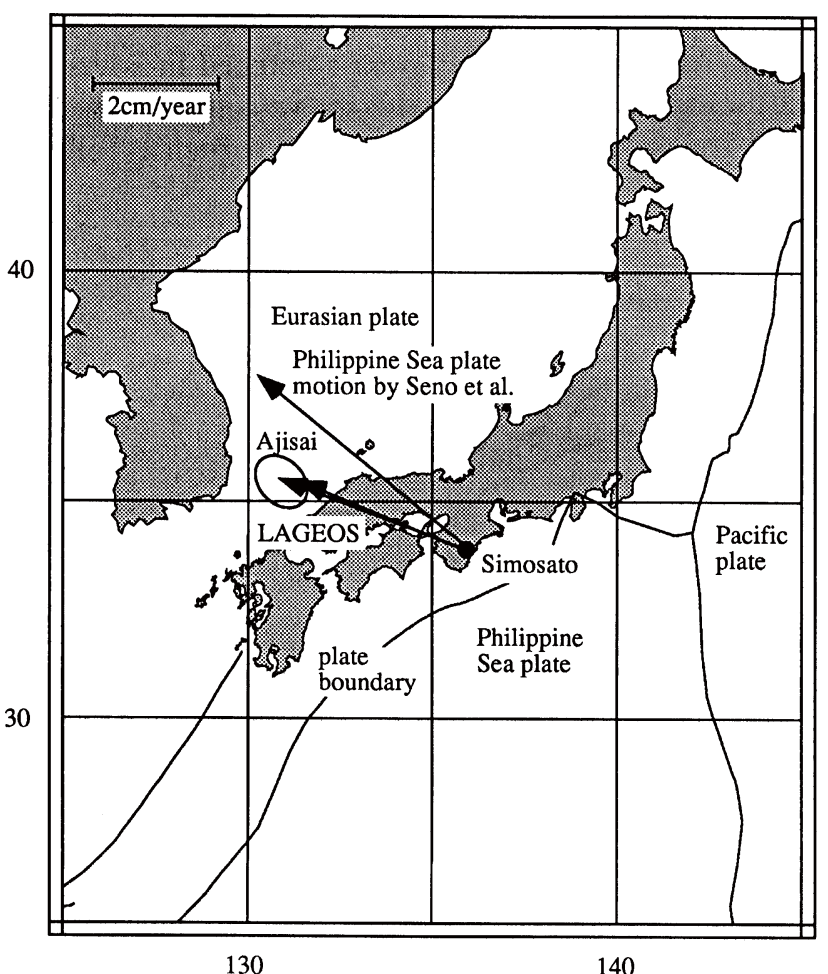

Fig. 10. The velocity of Simosato SLR station relative to the Eurasian NUVEL-1 plate motion determined by Ajisai. The LAGEOS result (SSC(CSR)94L01r02) and the Philippine Sea plate motion estimated from the Euler vector by Seno et al. (1993) are also shown.

$$
\begin{aligned}
-d l_{j k}= & d \dot{X}_{j}^{\mathrm{e}} \sin A_{j k}+d \dot{X}_{j}^{\mathrm{n}} \cos A_{j k} \\
& +d \dot{X}_{k}^{\mathrm{e}} \sin A_{k j}+d \dot{X}_{k}^{\mathrm{n}} \cos A_{k j}
\end{aligned}
$$

where $i_{j k}$ is the arc length rate between the $j$-th and $k$-th station in meter per year, $\dot{X}_{j}$ is the velocity of station $j$ in the Earth-fixed coordinate system in meter per year, and the superscripts e and $\mathrm{n}$ stand for eastward and northward components, respectively. Again, it should be noted that station velocity is projected on the sphere, not the ellipsoid. $A_{j k}$ is the azimuth angle of the arc from station $j$ to station $k$ at the station $j$ (see Fig. 9). For defining stations, Maui and Greenbelt, the following equations have been used in Eq. (6),

$$
d \dot{X}_{\text {Maui }}^{\mathrm{e}, \mathrm{n}}=0 \text { and } d \dot{X}_{\text {Greenbelt }}^{\mathrm{n}}=0
$$

Velocities of 23 stations, including the two defining

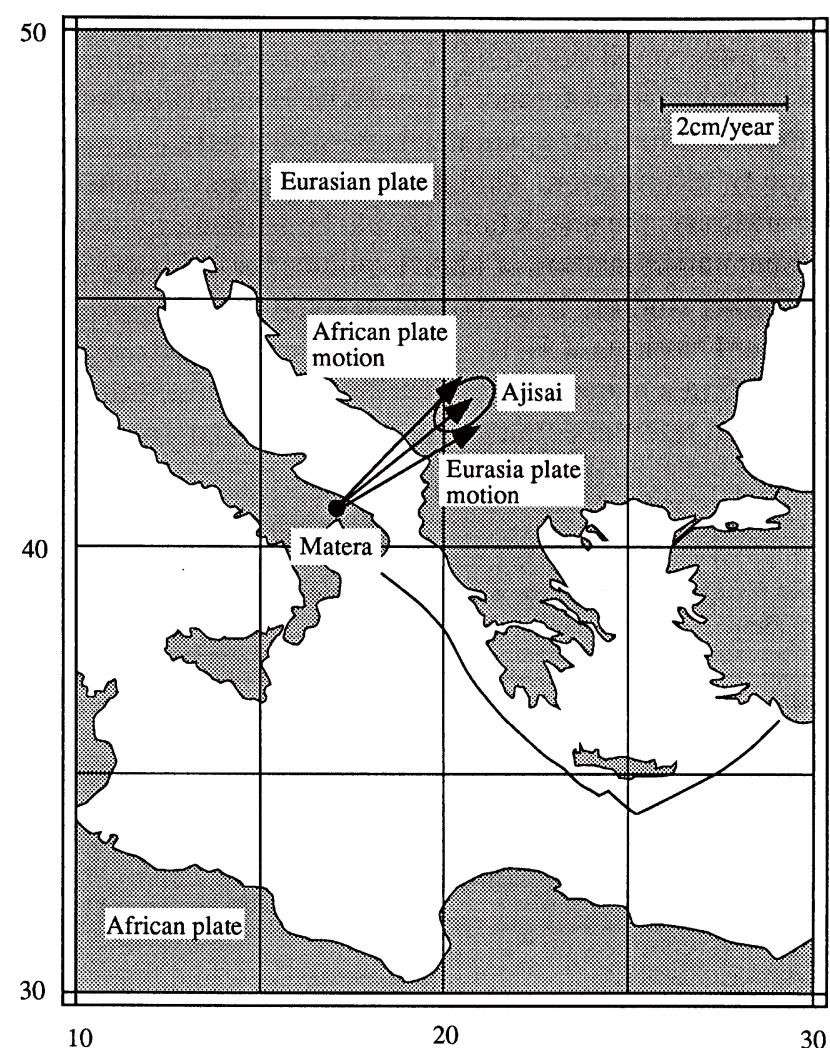

Fig. 11. The velocity of Matera in the NNR reference frame estimated by this study and the African/Eurasian NUVEL-1 plate motion.

stations, were recovered from 227 arc rates (Table 7). The reference velocity model was NUVEL-1 NNR. The semimajor and semiminor axis radii of error ellipses and orientation of the semimajor axis, measured counter-clockwise from the east, are also shown in Table 7. The velocities of Orroral, Huahine, Easter Island, San Fernando, Shanghai and Wettzell are poorly determined due to the short history of Ajisai tracking and/or sparse tracking data.

Figure 10 shows the velocity estimate and its error ellipse for the Simosato SLR station relative to the Eurasian NUVEL1 velocity determined by this study. The azimuth angle of the velocity is very close to that estimated from LAGEOS, but the velocity magnitude is slightly different. The discrepancy is within the uncertainty boundaries, however. The Philippine Sea plate motion estimated from the Euler vector by Seno et al. (1993) is also shown in the figure. Simosato is moving southeastward relative to the subducting plate. Simosato is located at the subduction zone near the Nankai Trough where the Philippine Sea plate subducts under the 
Eurasian plate. This location is known to have anomalous motion with respect to the Eurasian plate that might be caused by the collision of the plates (Sasaki, 1990; Smith et al., 1990; Robbins, et al., 1993; Robaudo and Harrison, 1993). The distance from Simosato to the Nankai Trough is about $100 \mathrm{~km}$ and the velocity of the subducting Philippine Sea plate with respect to the Eurasian plate is about $4-5 \mathrm{~cm} / \mathrm{yr}$ in this area. Hence, the discrepancy between the estimated and predicted arc length rates suggests the region is undergoing deformation in the plate boundary region. The amplitude of the estimated Simosato velocity is relatively close to that of the subducting plate, which implies that inter-plate coupling between the Eurasian and the Philippine Sea plate is strong in this region. Detailed geodetic surveys by the space geodetic techniques in this region will reveal the deformation mechanism at the plate boundary. Table 8 summarizes the results of velocity estimates for the Simosato station relative to the Eurasian NUVEL-1 plate motion.

The Matera station has been thought to be located on the Adriatic block, though its boundary is still not well known. The Adriatic block may be connected to the African plate (Channell et al., 1979), or an independent micro-plate (Mantovani et al., 1990). Smith et al. (1990) suggested the motion of Matera is slower than geologic predictions by using LAGEOS SLR data from 1978 to 1988 . Robbins et al. (1993) estimated the motion of Matera to be very close to the African NUVEL-1 motion from combined analysis of SLR and VLBI. Cenci et al. (1993) also concluded that the motion of Matera is similar to the African plate from the analysis of LAGEOS. Robaudo and Harrison (1993) estimated the motion of the Adriatic block by combining Matera and Medicina, a VLBI station in northern Italy. Based on a combination of SLR and GPS data, Noomen et al. (1996) found a deviation with respect to Africa motion of a mere 2 $\mathrm{mm} /$ year. Figure 11 shows the velocity estimates obtained by the author and the NUVEL-1 predictions for the Matera station. The estimated velocity is not purely Eurasian nor African, but somewhere between these velocities. Further Ajisai tracking data are needed to clarify the motion of Matera.

Bar Giyyora and Helwan are located near the plate boundary between the African and Arabian plates. The relative velocity of the plates at the boundary has been expected to be less than $1 \mathrm{~cm}$. Our study suggests the motions of these stations are more consistent with the African plate than the Arabian plate, but a deviation to the south exists, which is the opposite direction to the motion of the Arabian plate to the African plate (Fig. 12). The ITRF93 velocity model (Boucher et al., 1994) shows deviation of the Bar Giyyora motion from NUVEL-1, as large as $1 \mathrm{~cm} /$ year to the southeast from the African NUVEL-1 motion, but good agreement exists at Helwan.

Figure 13 is the velocity map for the European stations. The Eurasian NUVEL-1 plate motion is also shown. The intra-plate arcs in Europe seem to be stable. The estimated motion of Wettzell is quite unclear due to the short history of Ajisai tracking. Other stations agree well with the geologic prediction, except for Matera and Zimmerwald. Smith et al. (1990) reported a westward velocity anomaly of Zimmerwald station at the several $\mathrm{mm} /$ year level, which agrees well with this study. It should be noted that station velocity estimates in Europe may have a bias in the north-south direction due to poor geometry of the arc distribution in this direction.

Figure 14 shows the velocity estimates and the NUVEL1 motion for the Pacific SLR stations. It should be noted that the motion of Maui was fixed to the NUVEL-1 value. Yaragadee moves southward relative to the NUVEL-1 prediction, which agrees well with ITRF93, Smith et al. (1990)

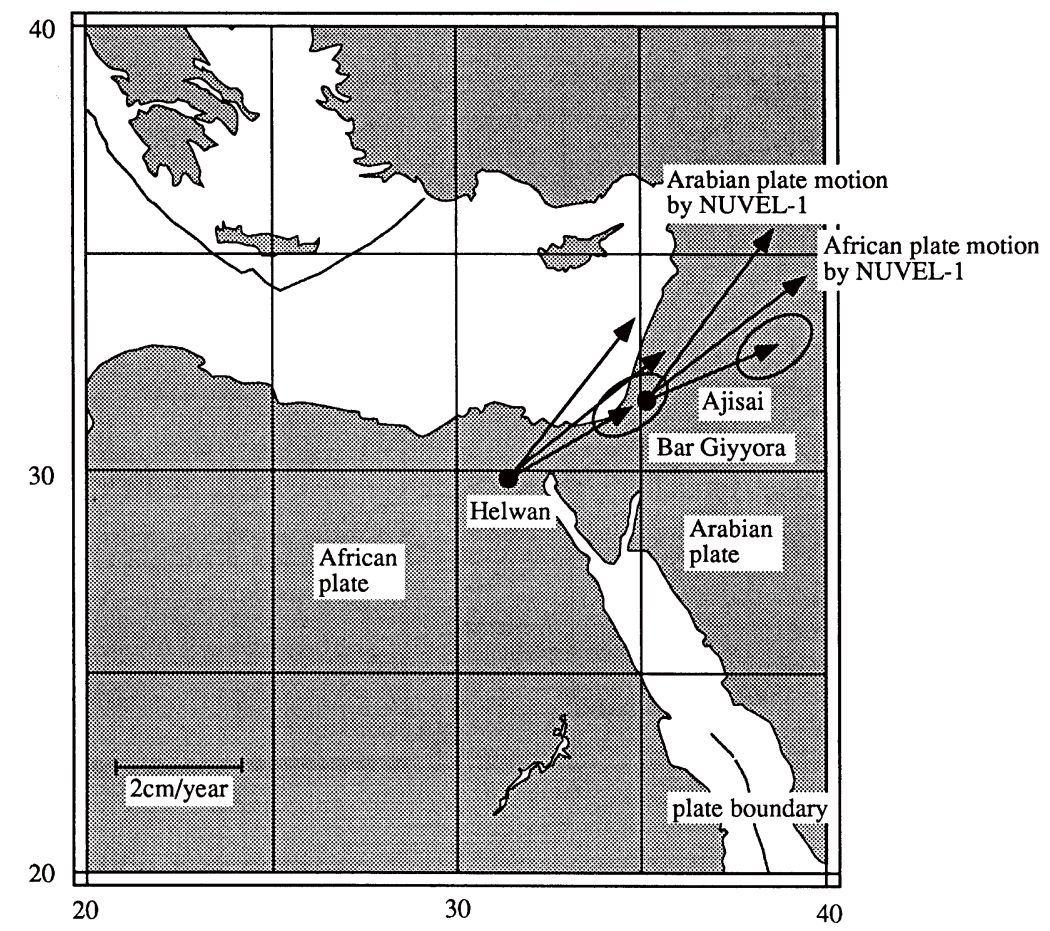

Fig. 12. The velocity of Helwan and Bar Giyyora in the NNR reference frame estimated by this study and the African/Arabian NUVEL-1 prediction. 


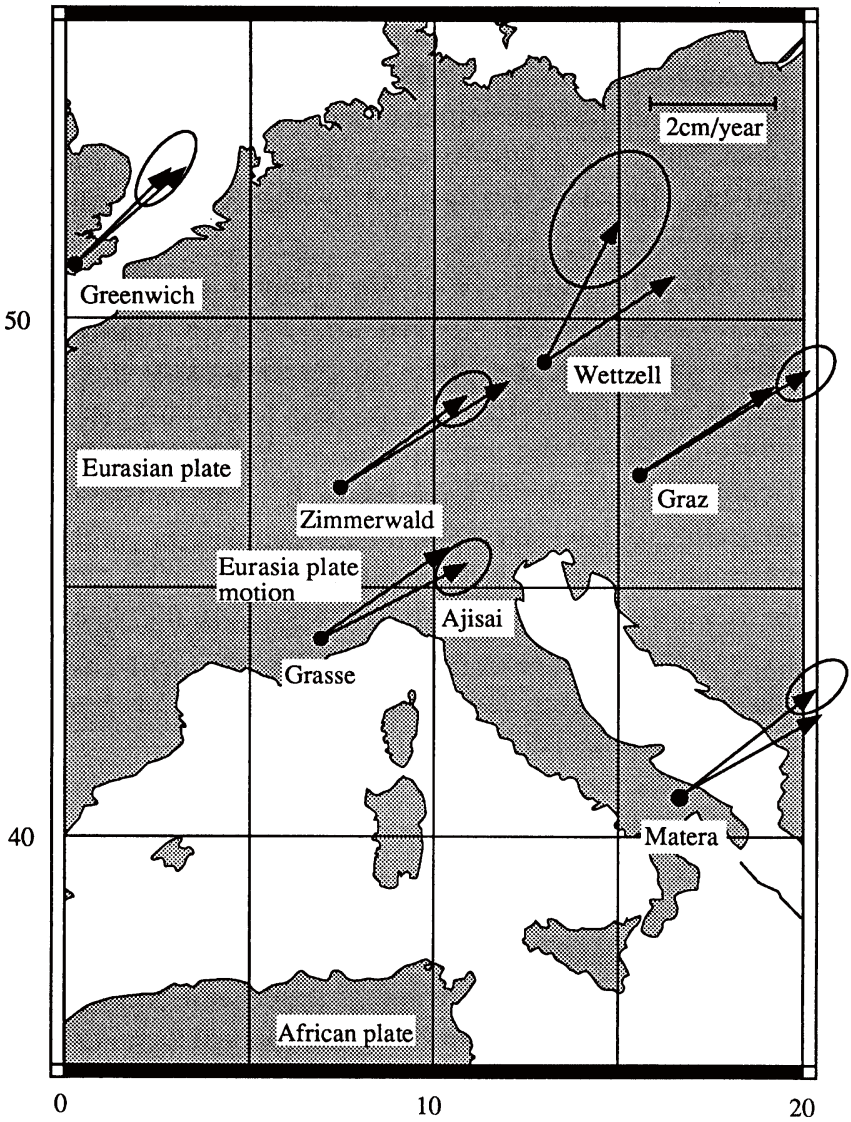

Fig. 13. The velocities of European SLR stations in the NNR reference frame determined by Ajisai SLR data and the Eurasian NUVEL-1 predictions. Arrows with error ellipses stand for the velocity estimated by this study. and Robbins et al. (1993), which implies that the predicted motion of the Australian plate by NUVEL-1 is too large. The motion of Orroral is not clear due to poor tracking coverage and unstable ranging observations. Shanghai seems to move eastward relative to the Eurasian NUVEL-1 motion with 3 $\mathrm{cm} /$ year, which may be caused by the stress within the Eurasian plate due to the collision of the Indian plate in northern India. ITRF93 reports eastward motion of Shanghai SLR station of $1 \mathrm{~cm} /$ year, though the analysis centers of IERS report various values for the Shanghai motion. The Shanghai SLR station has been recognized as a problematic station due to its variable data quality.

Figure 15 shows the velocity estimates of the North American SLR stations. The north-south component of the Greenbelt velocity was fixed to the NUVEL-1 value. The motions of the west coast stations in North America are affected by the neighboring plate motion, that is, the stations move in the direction of motion of the neighboring plate. However, the magnitude of the anomalous movement is very small, which implies low coupling at the plate boundary, especially around Monument Peak. The transition of site velocity between the North American and the Pacific motion does not happen abruptly, but occurs over a broad zone extending several hundreds of $\mathrm{km}$ in width (Ward, 1988). The difference between the geologically predicted and observed station velocities may reflect the degree of coupling at the plate boundary, the San Andreas Fault. Around Monument Peak, for example, the plate coupling may be lower than other regions. McDonald moves southeastward relative to the North American NUVEL-1 prediction.

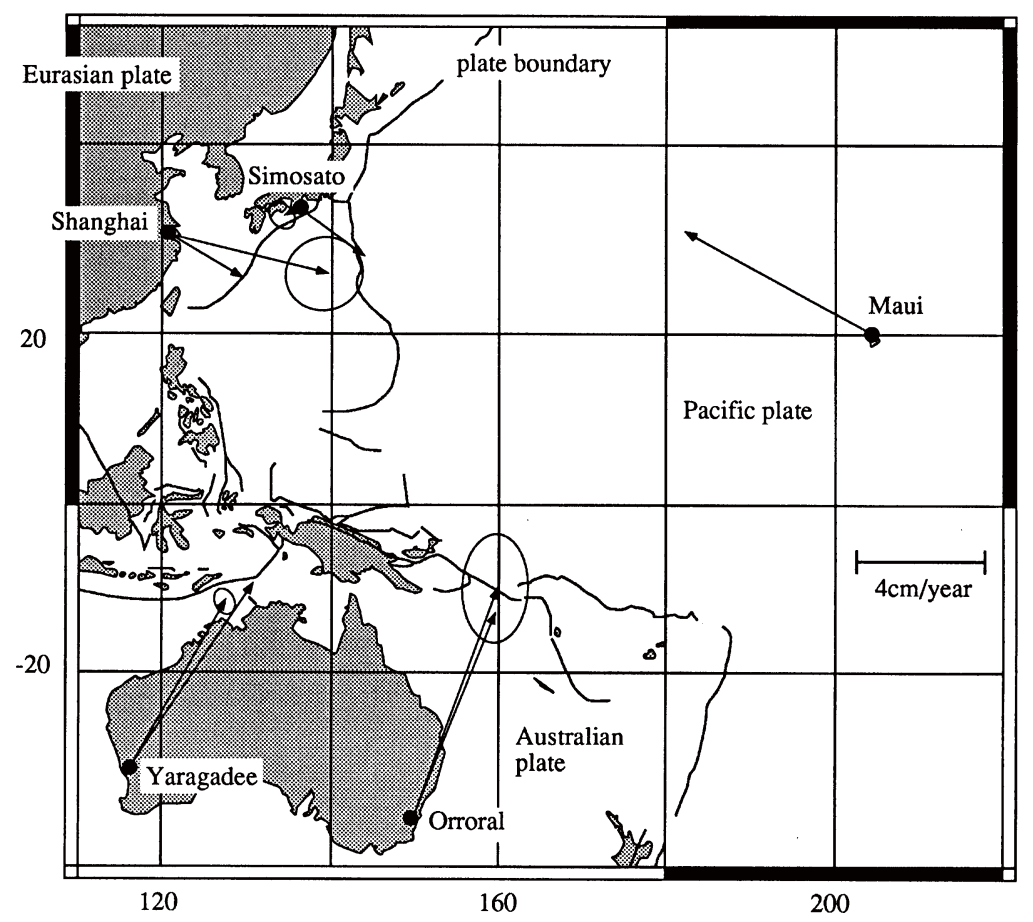

Fig. 14. The velocities of Pacific SLR stations in the NNR reference frame determined by Ajisai SLR data and the Eurasian/Australian/Pacific NUVEL1 predictions. Arrows with error ellipses stand for the velocity estimated by this study. 


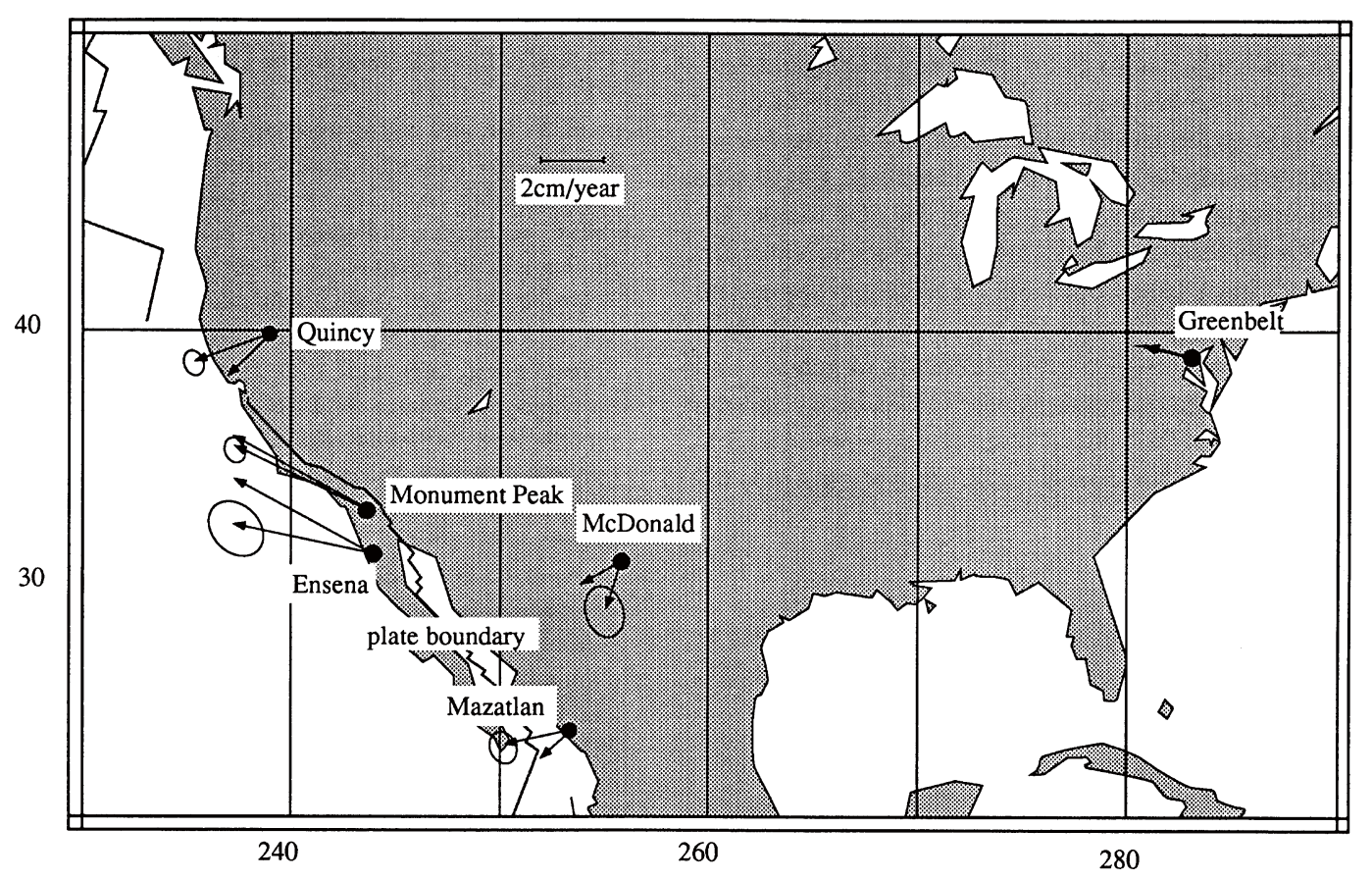

Fig. 15. The velocities of North American SLR stations in the NNR reference frame determined by Ajisai SLR data and the North American/Pacific NUVEL-1 predictions. Arrows with error ellipses stand for the velocity estimated by this study.

\section{Summary and Conclusions 5.1 Summary}

Eight years of Ajisai SLR data were analyzed year by year through a multi-arc approach with the UTOPIA software for the estimation of terrestrial reference frames and dynamical parameters. The stability of the geocenter determined by individual one-year arc analysis of Ajisai is $1 \mathrm{~cm}$. Consistency in the orientation and scale of the estimated terrestrial reference frame is typically 1 milli-arcsecond and $6 \times 10^{-9}$, respectively.

The estimated arc rates between stations well away from plate boundaries derived from Ajisai have strong correlation with the NUVEL-1A, ITRF93 and the CSR LAGEOS solution. Velocities of the observation stations were estimated by a weighted least squares method. The results were compared with geological predictions. Simosato moves northwestward with respect to the Eurasian plate that suggests plate boundary deformation. The amplitude of the estimated Simosato velocity is relatively close to the subducting plate velocity, which implies strong coupling between the two plates at the boundary. The motion of Matera is between the African and Eurasian plates. The analysis shows that movements of Bar Giyyora and Helwan deviate from geological predictions. Shanghai moves eastward relative to the Eurasian plate. The motions of the stations in the west coast of North America are affected by the neighboring plate motion.

\subsection{Conclusions}

Estimation of station velocities requires uniform data quality, which is crucially important for reliable estimates. SLR tracking technologies matured after the MERIT campaign in 1984, and the quality of Ajisai tracking data has been quite stable for most tracking stations since the launch of Ajisai. This stability is one of the advantages of the Ajisai SLR analysis. However, some stations with second genera- tion SLR equipments, such as Borowiec or Simeiz, have high noise. Improvement in these stations is very important for the study of the Eurasian intra-plate deformation that is inferred by the anomalous Shanghai motion.

It should be noted that an arc length and its rate are less affected by applied dynamical models and the combination of estimated parameters. However, experience has shown that $3 \mathrm{D}$ station positions are certainly dependent on these characteristics. For example, the geocenter difference described in Subsection 3.2 can be accentuated when other sets of parameters are estimated. Therefore, we must be cautious with the applied models and the analysis strategy if a stable TRF is required.

The range bias problem in Ajisai SLR tracking data needs to be resolved in order to accurately determine the ellipsoidal height of ground stations from Ajisai data. However, it should be noted that this problem does not affect the results of horizontal station motion.

This result shows availability of Ajisai for plate motion studies. Combining Ajisai SLR data with LAGEOS, LAGEOS-2, STARLETTE and STELLA will enhance our understanding of plate tectonics as well as Earth rotation, geopotential field and ocean tide modelings. Ajisai is a good target for weak SLR stations, hence, it provides dense tracking data at global SLR stations.

The SLR technique will play an important role in determination of the geocenter, terrestrial reference frames and tectonic motions. Combination of several geodetic satellites will surely enhance our knowledge of geodesy and geophysics. SLR will provide an absolute reference for sea level monitoring, and continuous SLR observation will improve Earth's gravity field and its variation, which will contribute to studies of the solid Earth-atmosphere-ocean system. 
Acknowledgments. The author gratefully acknowledges the kind support and precious suggestions by Dr. Minkang Cheng and Prof. Robert E. Schutz during his stay in the University of Texas at Austin. He wishes to appreciate Prof. R. E. Schutz who brushed up the paper. He acknowledges Drs. R. Noomen and E. C. Pavlis who carefully and thoroughly reviewed the paper. He also thanks Dr. Minoru Sasaki for his long-standing support and appreciates Dr. Masayuki Fujita for his helpful comment. He wishes to appreciate Dr. Toshihiro Kubo-oka for his help. He expresses his gratitude to the personnel of the global SLR tracking network in general and the members of the Simosato Hydrographic Observatory and the Geodesy and Geophysics Division of the Hydrographic Department of Japan in particular for their great effort to track Ajisai for years.

\section{References}

Aoki, S., B. Guinot, G. H. Kaplan, H. Kinoshita, D. D. McCarthy, and P. K. Seidelmann, The new definition of universal time, Astron. Astrophys. 105, 359-361, 1982.

Argus, D. F. and G. A. Lyzenga, Constraints on interseismic deformation at Japan Trench from VLBI data, Geophys. Res. Lett., 20, 611-614, 1993.

Barlier, R., C. Berger, J. Falin, G. Kockarts, and G. Thuillier, Atmospheric model based on satellite drag data, Ann. Geophys., 34, 9-24, 1978.

Boucher, C., Z. Altamimi, and L. Duhem, Results and Analysis of the ITRF93, IERS Technical Note 18, Observatoire de Paris, Paris, 1994

Cenci, A., M. Fermi, C. Sciarretta, R. Devoti, and A. Caporali, Tectonic Motion in the Mediterranean Area from Laser Ranging to LAGEOS, in Contribution of Space Geodesy to Geodynamics: Crustal Dynamics, edited by D. E. Smith and D. L. Turcotte, pp. 347-358, Geodynamics Series, AGU, Washington, D.C., U.S.A., 1993.

Channell, J. E. T., B. D'Argenio, and F. Horvath, Adria, the African promontory, Mesozoic Mediterranean paleogeography, Earth Sci. Rev., 15, 213-292, 1979.

Cheng, M. K., R. J. Eanes, C. K. Shum, B. E. Schutz, and B. D. Tapley, Ocean tide model for satellite orbit determination, The 12th International Symposium on Earth Tides, Beijing, China, Aug. 4, 1993.

Christodoulidis, D. C., D. E. Smith, R. Kolenkiewicz, S. M. Klosko, M. H. Torrence, and P. J. Dunn, Observing tectonic plate motions and deformations from satellite Laser Ranging, J. Geophys. Res., 90, 92499263, 1985

DeMets, C., R. G. Gordon, D. F. Argus, and S. Stein, Current plate motions, Geophys. J. Int., 101, 425-478, 1990.

DeMets, C., R. G. Gordon, D. F. Argus, and S. Stein, Effect of recent revisions to the geomagnetic reversal time scale on estimates of current plate motions, Geophys. Res. Lett., 21, 2191-2194, 1994.

Dixon, T. H., GPS measurement of relative motion of the Cocos and Caribbean plates and strain accumulation across the middle America trench, Geophys. Res. Lett., 20, 2167-2170, 1991.

Eanes, R. J. and M. M. Watkins, Earth orientation and site coordinates from the Center for Space Research Solution, Earth orientation, reference frames and atmospheric excitation functions submitted for the 1993 IERS Annual report, IERS Tech. Note 17, Central Bureau of IERS, Observatoire de Paris, Paris, France, 1994

Fallon, F. W. and W. H. Dillinger, Crustal velocities from geodetic very long baseline interferometry, J. Geophys. Res., 97, 7129-7136, 1992

Feigl, K. L., D. C. Agnew, Y. Bock, D. Dong, A. Donnellan, B. H. Hager, T. A. Herring, D. D. Jackson, T. H. Jordan, R. W. King, S. Larsen, K M. Larson, M. H. Murray, Z. Shen, and F. H. Webb, Space Geodetic Measurement of Crustal Deformation in Central and Southern California, 1984-1992, J. Geophys. Res., 98, 21677-21712, 1993.

Gordon, D., C. Ma, and J. W. Ryan, Results from the CDP Mobile VLBI Program in the Western United States, in Crustal Dynamics, Contributions of Space Geodesy to Geodynamics, edited by D. E. Smith and D. L. Turcotte, Geodynamics Series, 23, pp. 131-138, American Geophysical Union, Washington, D.C., 1993.

Harrison, C. G. A. and N. B. Douglas, Satellite Laser Ranging and geological constraints on plate motions, Tectonics, 9, 935-952, 1990

Holdridge, D. B., An Alternate Expression for Light Time Using General Relativity, JPL Space Program Summary 37-48, III, 2-4, Jet Propulsion Laboratory, NASA, Pasadena, CA, U.S.A., 1967.
Knocke, P. and J. C. Ries, Earth radiation pressure effects on satellites, Center for Space Research, The University of Texas at Austin, Austin, TX, U.S.A., 1987.

Larson, K. M. and J. Freymueller, Relative motions of the Australian, Pacific, and Antarctic plates using the Global Positioning System, Geophys. Res. Lett., 22, 37-40, 1995.

Lerch, F., R. Nerem, B. Putney, T. Felstentreger, B. Sanchez, S. Klosko, G. Patel, R. Williamson, D. Chinn, J. Chan, K. Rachlin, N. Chandler, J. McCarthy, J. Marshall, S. Luthcke, D. Pavlis, J. Robbins, S. Kapoor, and E. Pavlis, NASA Tech. Memo. 104555, NASA Goddard Space Flight Center, Greenbelt, MD, 1992.

Lieske, J. H., T. Lederle, W. Fricke, and B. Morando, Expression for the precession quantities based upon the IAU (1976) system of astronomical constants, Astron. Astrophys., 58, 1-16, 1977.

Lisowski, M., Recent plate motions and crustal deformation, Rev. Geophys., 29, 162-171, 1991

Mantovani, E., D. Babbucci, D. Albarello, and M. Mucciarelli, Deformation pattern in the central Mediterranean and behavior of the African/ Adriatic promontory, Tectonophysics, 179, 63-79, 1990.

Marini, J. W. and C. W. Murray, Jr., Correction of laser range tracking data for atmospheric refraction at elevation above 10 degrees, Rep. X591-73-351, Goddard Space Flight Center, NASA, Greenbelt, MD, November 1973.

McCarthy, D. D. (ed.), IERS Standards (1992), Central Bureau of IERS, Observatoire de Paris, Paris, France, 1992.

McMillan, J. D., Mathematical Specifications of the University of Texas Orbit Processor and Application to the Laser Observations of the Beacon Explorer Satellite, AMRL 1052, Applied Mechanics Laboratory, The University of Texas at Austin, Austin, TX, U.S.A., 1973.

Minster, J. B. and T. H. Jordan, Present-day plate motions, J. Geophys. Res., 83, 5331-5354, 1978.

Newhall, X. X., E. M. Standish, and J. G. Williams, DE-102, a numerically integrated ephemeris of the moon and the planets spanning fortyfour centuries, Astron. Astrophys., 125, 150-167, 1983.

Noomen, R., B. A. C. Ambrosius, and K. F. Wakker, Crustal motions in the Mediterranean Region determined from Laser Ranging to LAGEOS, in Crustal Dynamics, Contributions of Space Geodesy to Geodynamics, edited by D. E. Smith and D. L. Turcotte, Geodynamics Series, 23, pp. 331-346, American Geophysical Union, Washington, D.C., 1993.

Noomen, R., T. A. Springer, B. A. C. Ambrosius, K. Herzberger, D. C. Kuijper, G.-J. Mets, B. Overgaauw, and K. F. Wakker, Crustal deformations in the Mediterranean area computed from SLR and GPS observations, J. Geodyn., 21, 73-96, 1996.

Press, W. H., S. A. Teukolsky, W. T. Vetterling, and B. P. Flannery, Numerical Receipes, 2nd ed., 653 pp., Cambridge Univ. Press, N.Y., U.S.A., 1986.

Ries, J. C., C. Huang, and M. M. Watkins, Effect of general relativity on a near-Earth satellite in the geocentric and barycentric reference frames, Phys. Rev. Lett., 61, 903-906, 1988.

Robaudo, S. and C. G. A. Harrison, Plate Tectonics from SLR and VLBI global data, in Contributions of Space Geodesy to Geodynamics: Crustal Dynamics, edited by D. E. Smith and D. L. Turcotte, pp. 5171, American Geophysical Union, Washington, D.C., U.S.A., 1993.

Robbins, J. W., D. E. Smith, and C. Ma, Horizontal crustal deformation and large scale plate motions inferred from space geodetic techniques, in Crustal Dynamics, Contributions of Space Geodesy to Geodynamics, edited by D. E. Smith and D. L. Turcotte, Geodynamics Series, 23, pp. 21-36, American Geophysical Union, Washington, D.C., 1993.

Ryan, J. W., T. A. Clark, C. Ma, D. Gordon, D. S. Caprette, and W. E. Himwich, Global scale tectonic plate motions measured with CDP VLBI data, in Crustal Dynamics, Contributions of Space Geodesy to Geodynamics, edited by D. E. Smith and D. L. Turcotte, Geodynamics Series, 23, pp. 37-49, American Geophysical Union, Washington, D.C., 1993a.

Ryan, J. W., C. Ma, and D. S. Caprette, NASA space geodesy programGSFC data analysis-1992, NASA Tech. Memo., 104572, 471, 1993 b.

Sasaki, M., Study of the earth's dynamics by means of satellite laser ranging techniques, Rep. Hydrogr. Res., 26, 99-187, 1990.

Sasaki, M. and H. Hashimoto, Launch and observation program of the experimental geodetic satellite of Japan, IEEE Transactions on Geoscience and Remote Sensing, GE-25, 5, 526-533, 1987.

Sasaki, M. and A. Sengoku, SLR observation and data analysis made by 
the Hydrographic Department of Japan in the last decade and the motion of the Simosato site, Proc. of the International Workshop for Reference Frame Establishment and Technical Development in Space Geodesy (iRiS '93 TOKYO), pp. 134-141, Communications Research Lab., Tokyo, Jan. 18-21, 1993.

Saucier, F. and E. Humphreys, Horizontal crustal deformation in Southern California from joint models of geologic and very long baseline interferometry measurements, in Crustal Dynamics, Contributions of Space Geodesy to Geodynamics, edited by D. E. Smith and D. L. Turcotte, Geodynamics Series, 23, pp. 139-176, American Geophysical Union, Washington, D.C., 1993.

Sengoku, A., Determination of the precise positions of Titi Sima and Isigaki Sima by satellite laser ranging, Rep. Hydrogr. Res., 27, 181195, 1991.

Sengoku, A., Estimation of the positions of the first order control points from global analysis of Ajisai and LAGEOS SLR data, Rep. Hydrogr. Res., 33, 53-60, 1997.

Sengoku, A., M. Cheng, and B. E. Schutz, Anisotropic reflection effect on satellite, Ajisai, J. Geod., 70, 140-145, 1995.

Seno, T., S. Stein, and A. E. Gripp, A model for the motion of the Philippine Sea plate consistent with NUVEL-1 and geological data, $J$. Geophys. Res., 98, 17941-17948, 1993.

Sinclair, A. T. and G. M. Appleby, A short-arc method for determination of station coordinates and baselines applied to the Mediterranean Area, in Crustal Dynamics, Contributions of Space Geodesy to Geodynamics, edited by D. E. Smith and D. L. Turcotte, Geodynamics Series, 23, pp. 389-396, American Geophysical Union, Washington, D.C., 1993.

Smith, D. E., R. Kolenkiewicz, P. J. Dunn, and M. H. Torrence, The measurement of fault motion by satellite laser ranging, Tectonophysics, 52, 59-67, 1979.

Smith, D. E., R. Kolenkiewicz, P. J. Dunn, J. W. Robbins, M. H. Torrence, S. M. Klosko, R. G. Williamson, E. C. Pavlis, N. B. Douglas, and S. K Fricke, Tectonic motion and deformation from satellite laser ranging to LAGEOS, J. Geophys. Res., 95, 22013-22041, 1990.

Smith D. E., R. Kolenkiewicz, P. J. Dunn, S. M. Klosko, J. W. Robbins, M. H. Torrence, R. G. Williamson, E. C. Pavlis, N. B. Douglas, and S. K. Fricke, LAGEOS Geodetic Analysis-SL7.1, NASA Tech. Memo. 104549, NASA, 1991.

Smith, D. E., J. W. Robbins, and C. Ma, Large-scale plate behavior and horizontal crustal deformation inferred from space geodetic tech- niques, International Workshop on Global Positioning System in Geosciences, Crete, 1992.

Smith, D. E., R. Kolenkiewicz, J. W. Robbins, P. J. Dunn, M. H. Torrence, R. G. Williamson, and M. B. Heflin, Kinematics of Deformation Zones Determined by Space Geodesy, XXI IUGG General Assembly, Boulder, CO, U.S.A., 1995.

Tapley, B. D., B. E. Schutz, and R. J. Eanes, Station coordinates, baselines, and Earth rotation from LAGEOS Laser Ranging: 19761984, J. Geophys. Res., 90, 9235-9248, 1985.

Tapley, B. D., M. M. Watkins, J. C. Ries, G. W. Davis, R. J. Eanes, S. R. Poole, H. J. Rim, B. E. Schutz, C. K. Shum, R. S. Nerem, F. J. Lerch, E. Pavlis, S. M. Klosko, N. K. Pavlis, and R. G. Williamson, The JGM3 Gravity Model, XIX General Assembly of the European Geophysical Society, Grenoble, France, April 25-29, 1994

Tsuji, H., Detection of plate motions around Japan based on daily GPS measurements, J. Geod. Soc. Japan, 41, 47-73, 1995.

Wahr, J. M., The forced nutations of an elliptical, rotating, elastic, and oceanless Earth, Gephys. J. Roy. Astron. Soc., 64, 705-727, 1981.

Ward, S. N., North-America-Pacific plate boundary, and elastic-plastic megashear: Evidence from very long baseline interferometry, $J$. Geophys. Res., 93, 7716-7728, 1988.

Watkins, M. M., Tracking station coordinates and their temporal evolution as determined from laser ranging to the LAGEOS satellite, CSR90-1, Center for Space Research, The University of Texas at Austin, Austin, TX, U.S.A., 1990.

Wessel, P. and W. H. F. Smith, Free software helps map and display data, EOS Trans. Amer. Geophys. U., 72, 441, 445-446, 1991.

Wilson, P. and E. Reinhart, The Wegener-Medlas Project preliminary results on the determination of the geokinematics of the Eastern Mediterranean, in Crustal Dynamics, Contributions of Space Geodesy to Geodynamics, edited by D. E. Smith and D. L. Turcotte, Geodynamics Series, 23, pp. 299-309, American Geophysical Union, Washington, D.C., 1993.

Zerbini, S., Crustal motions from short-arc analysis of LAGEOS data, in Crustal Dynamics, Contributions of Space Geodesy to Geodynamics, edited by D. E. Smith and D. L. Turcotte, Geodynamics Series, 23, pp. 371-387, American Geophysical Union, Washington, D.C., 1993.

A. Sengoku (e-mail: asengoku@cue.jhd.go.jp) 